\title{
Quantum coherence and lifetimes of surface-state electrons
}

\author{
L. Bürgi ${ }^{a}, H$. Brune $^{\mathrm{a}, *}$, O. Jeandupeux ${ }^{\mathrm{a}}, \mathrm{K} . \mathrm{Kern}^{\mathrm{b}}$ \\ ${ }^{a}$ Institut de Physique Expérimentale, Ecole Polytechnique Fédérale de Lausanne, CH-1015 Lausanne, Switzerland \\ ${ }^{\mathrm{b}}$ Max-Planck-Institut für Festkörperforschung, Heisenbergstrasse 1, D-70569, Stuttgart, Germany
}

Received 23 October 1999; received in revised form 17 December 1999; accepted 17 December 1999

\begin{abstract}
We discuss a novel approach to measure the electron phase-relaxation length and femtosecond lifetimes at surfaces. It relies on the study of the spatial decay of quantum interference patterns in the local density of states (LDOS) with the STM. The method has been applied to $\mathrm{s}-\mathrm{p}$ derived surface-state electrons on $\mathrm{Cu}(111)$ and $\mathrm{Ag}(111)$. The characteristic decay length of the LDOS oscillations is influenced by the finite lifetime, and thus reveals information about inelastic scattering in the two-dimensional (2D) electron gas. After an introduction in Section 1, we present a model describing the decay of Friedel oscillations off from straight steps in Section 2. Energy dependent lifetime measurements of hot electrons are presented in Section 3 and interpreted in terms of electron-electron scattering. The temperature dependent lifetime measurements of low-energy quasiparticles discussed in Section 4 give insight into the interaction of these 2D electrons with phonons. Our results on inelastic lifetimes are discussed in comparison with high-resolution angle-resolved photoemission and femtosecond two-photon photoemission measurements. (C) 2000 Elsevier Science B.V. All rights reserved.
\end{abstract}

Keywords: Electron phase-relaxation length; Quantum interference patterns; Scanning tunneling microscopy-spectroscopy; Surface states

\section{Introduction}

The phase-relaxation length $L_{\phi}$, i.e. the distance a quasiparticle can propagate without loosing its phase memory, is a key quantity in solid state physics. Quantum mechanical interference phenomena can only prevail if $L_{\phi}$ is larger than any other relevant length scale [1]. Examples include Aharonov-Bohm oscillations, quantum Hall effect, Friedel oscillations and localization. With respect to surface physics $L_{\phi}$,

*Corresponding author. Tel.: +41-21-693-5451; fax: +41-21693-3604.

E-mail address: harald.brune@ipe.dp.epfl.ch (H. Brune). or equivalently the lifetime $\tau_{\phi}$ of the quasiparticle ${ }^{1}$, is of particular interest, since it governs the dynamics of charge transfer and electronic excitations in surface chemistry [2]. Also, a sufficiently long $L_{\phi}$ is a prerequisite for the standing waves to appear.

Collisions of an electron with static scatterers, i.e. scatterers with no internal degree of freedom, do not influence the phase coherence [1]. On the other hand, $L_{\phi}$ is reduced by inelastic scattering processes like electron-phonon $(\mathrm{e}-\mathrm{ph})$ or electron-electron $(\mathrm{e}-\mathrm{e})$

\footnotetext{
${ }^{1}$ If $L_{\mathrm{M}}>L_{\phi}$, then $\tau_{\phi}=L_{\phi} / v$ where $v$ is the group velocity of the electron [1]. $L_{\mathrm{M}}$ is the elastic mean free path of a surface-state electron, i.e., the mean distance between static surface scatterers such as steps or adsorbates.
} 
interaction. To familiarize with the order of magnitude of typical lifetimes in metals, we discuss in this introduction the e-e and e-ph scattering rates of a quasiparticle of energy $E-E_{\mathrm{F}}$ with respect to the Fermi sea in simple models. Fermi liquid theory (FLT) for a 3D free electron gas predicts the following energy dependence of the e-e lifetime at $T=0 \mathrm{~K}\left(E-E_{\mathrm{F}} \ll E_{0}\right)[3,4]:$

$$
\begin{aligned}
& \tau_{\mathrm{e}-\mathrm{e}}(E)=\tau_{0}\left(\frac{E_{0}}{E-E_{\mathrm{F}}}\right)^{2}, \\
& \tau_{0}=\frac{64}{\pi^{2} \sqrt{3 \pi}} \sqrt{\frac{m_{e}}{n e^{2}}},
\end{aligned}
$$

where $n$ is the density of the electron gas and $E_{0}$ the width of the band. e-e processes at low excitation energies are dominated by electron-hole pair creation, and the inverse quadratic excess energy dependence basically relies on a phase space argument, i.e. the larger the initial excess energy $E-E_{\mathrm{F}}$ the more final states with an additional electron-hole pair are accessible [5]. In FLT the temperature dependence of $\tau_{\mathrm{e}-\mathrm{e}}$ for electrons at $E_{\mathrm{F}}$ is given by

$$
\tau_{\mathrm{e}-\mathrm{e}}(T)=\tau_{0}\left(\frac{E_{0}}{\pi k_{\mathrm{B}} T}\right)^{2} .
$$

The e-ph scattering rate can be estimated within a Debye model [6,7]:

$$
\begin{aligned}
\frac{\hbar}{\tau_{\mathrm{e}-\mathrm{ph}}(E, T)}= & 2 \pi \hbar \int_{0}^{\omega_{\mathrm{D}}} \mathrm{d} \omega^{\prime} \lambda \\
& \cdot\left(\frac{\omega^{\prime}}{\omega_{\mathrm{D}}}\right)^{2}\left(1-f\left(E-\hbar \omega^{\prime}, T\right)\right. \\
& \left.+b\left(\hbar \omega^{\prime}, T\right)+f\left(E+\hbar \omega^{\prime}, T\right)\right) .
\end{aligned}
$$

Here $\omega_{\mathrm{D}}$ is the Debye frequency, $\lambda$ the electronphonon mass enhancement parameter and $b(E, T)$ the Bose-Einstein distribution function ${ }^{2}$. One readily shows that

$$
\frac{\hbar}{\tau_{\mathrm{e}-\mathrm{ph}}\left(E_{\mathrm{F}}, T\right)}=2 \pi \lambda k_{\mathrm{B}} T
$$

when $k_{\mathrm{B}} T \gg \hbar \omega_{\mathrm{D}}$. For any $E$ and $T$ the integral in Eq. (3) has to be calculated numerically. Fig. 1

\footnotetext{
${ }^{2}$ Remember that energies are always with respect to $E_{\mathrm{F}}$.
}

shows the calculated lifetimes for bulk $\mathrm{Cu}$. The e-ph scattering rate is independent of energy as long as $E-E_{\mathrm{F}}>\hbar \omega_{\mathrm{D}}$ (dashed line in Fig. 1(a)). It is clear from Fig. 1(a) that e-e scattering dominates e-ph scattering at low temperatures and large excess energies $(\geq 0.5 \mathrm{eV})$, whereas at energies very close to the Fermi level inelastic scattering is dominated by e-ph processes at all temperatures of interest ${ }^{3}$.

Elegant experiments have been performed to determine $L_{\phi}$ of ballistic electrons in low-density high-mobility two-dimensional electron gases present at the interfaces of semiconductor heterostructures. In particular, the excess energy and temperature dependence of $L_{\phi}$ in GaAs/AlGaAs heterostructures has been measured by Yacoby et al. [11] and Murphy et al. [12], respectively, where the main contribution to $L_{\phi}$ could be attributed to electron-electron (e-e) scattering, in striking agreement with Fermi liquid theory for a 2D electron gas (2DEG) [13,14]. Another access to electron and hole lifetimes (and hence to $L_{\phi}$ ) has become possible through electron spectroscopic methods on single-crystal metal surfaces $[15,16]$. In particular the photohole lifetimes of noble-metal surface states have been investigated with high-resolution angle-resolved photoemission (ARPES), revealing Lorentzian line shapes [7,10,1518], whose full peak widths at half maximum (FWHM) $\Gamma$ give access to the lifetime via $\Gamma=\hbar / \tau_{\phi}$. Although the phonon contribution to copper surfacestate lifetimes has been successfully determined with ARPES [7,10], the assignment of ARPES-linewidths to true quasiparticle lifetimes is complicated by nonlifetime effects $[19,20]$, e.g. due to impurities, and hence the absolute values of ARPES-lifetimes have to be considered as lower limits [7]. Furthermore, recent femtosecond time-resolved two-photon photoemission (2PPE) experiments opened up a new path to measure excess energy dependent lifetimes of hot bulk quasiparticles for metals and semimetals [2128]. However, due to cascade and depopulation effects the interpretation of 2PPE spectra is a difficult task, and up to now it does not seem to be

\footnotetext{
${ }^{3}$ The above estimates were derived for free-like $(s p)$ electrons and generally do not apply to $d$-electrons. The lifetime of $\mathrm{Cu} d$ electrons at the top of the $d$-band $(E=-2 \mathrm{eV})$ is comparatively long $[8,9]$ and e-ph scattering can be important [10].
} 

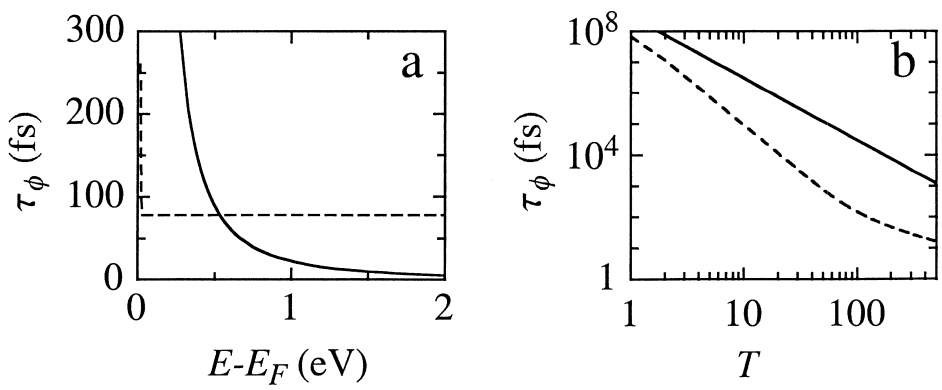

Fig. 1. e-e (full line) and e-ph (dashed line) lifetimes as calculated using 3D Fermi liquid theory (Eqs. (1) and (2)) and a Debye model (Eq. (3)), respectively, for $\mathrm{Cu}$ parameters: $\tau_{0}=0.46 \mathrm{fs}, E_{0}=7 \mathrm{eV}, \omega_{\mathrm{D}}=27 \mathrm{meV}, \lambda=0.15$ [5,6]. (a) Lifetime at $T=0 \mathrm{~K}$ as a function of excess energy of the quasiparticle with respect to the Fermi sea. (b) Temperature dependence of the lifetimes for particles at the Fermi level (double logarithmic plot).

clarified why different groups report lifetimes which vary by up to a factor of 4 for the very same system $[23,27]$.

In the field of STM many authors have qualitatively discussed a possible contribution of quasiparticle and electron-phonon interactions to the damping of interference patterns and to spectroscopic linewidth [29-35]. Especially, Crampin and Bryant emphasized the importance of quasiparticle interactions to interpret the spectroscopic linewidth of confined electrons in quantum corrals [36]. However, it was only recently that $\mathrm{Li}$ et al. used STM to determine the lifetime of excited holes at the band edge of the $\operatorname{Ag}(111)$ surface state quantitatively $[37,38]$. Similar to ARPES, Li et al. have investigated the linewidth of the surface-state onset in tunneling spectra. The advantage over ARPES is the capability to choose a surface spot bare of impurities, and hence non-lifetime effects are reduced. However, with the method used by Li et al., $L_{\phi}$ of excited holes can be studied only at a single energy, namely $E_{\Gamma}^{-}$, which constitutes a major limitation. In Ref. [38] Li et al. state that the width of the surface-state onset provides a local measure of surface-state lifetimes. Note that this is only correct if static scatterers are absent. Interference effects due to the presence of such scatterers can lead to a substantial broadening of the surface-state onset, which is a pure nonlifetime effect. This is illustrated in Fig. 2, where the LDOS in the presence of a straight step edge, $\rho(E)=$ $L_{0}\left(1-J_{0}\left(2 k_{\mathrm{E}} x\right)\right.$ ) (see Eq. (10) below for a step reflection amplitude of $r=1$ ), is plotted for different distances $x$ from the step. Far away from the scatterer the onset is indeed infinitely sharp due to the absence of inelastic processes. But close to the step edge the onset is substantially broadened because of interference effects. Note that in our case of $r=1$ there is no contribution from the surface state at $x=0$, due to the fact that all surface-state wave functions have to vanish at the (hard wall) step edge location. The reduction of the LDOS at and close to scattering centers $[29,38,39]$ is thus imposed by the potential of the scatterer and can be understood in the framework of the simplest model.

In this contribution we present a new approach to measure lifetimes of surface-state and surface-resonance electrons locally with an STM. (The term surface states shall include surface resonances.) To do so we have studied the decay of LDOS interference patterns of surface-state electrons scattering off descending straight step edges; the decay is influenced by the loss of coherence and hence by $L_{\phi}$. The major interest to do lifetime measurements with

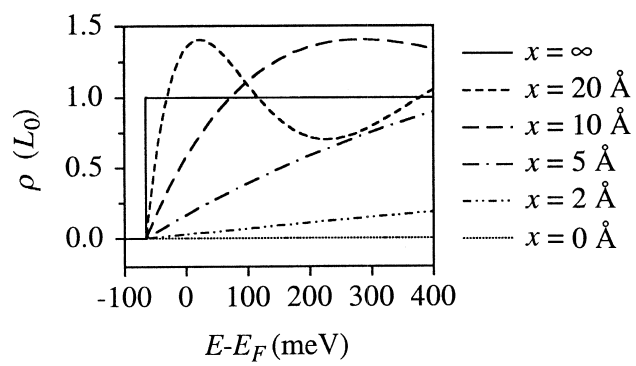

Fig. 2. DOS at different distances from a straight step edge modeled as infinite square barrier $\left(\operatorname{Ag}(111)\right.$ parameters: $E_{\Gamma}^{-}=$ $-65 \mathrm{meV}, m^{*}=0.4 m_{e}$ ). 
an STM comes from the fact that STM offers a complete control over impurities, i.e. surface spots bare of defects can be chosen for the lifetime measurements, and thus non-lifetime effects, embarrassing all integral techniques like ARPES and $k$ resolved inverse photoelectron spectroscopy (KRIPES), may be completely avoided.

\section{Model}

In the following we present a model that gives a quantitative description of the decay of LDOS oscillations away from straight steps. We will first derive and test the model in the absence of inelastic scattering on the terrace $\left(L_{\phi}=\infty\right)$, we then include inelastic scattering events and quantify the additional damping giving access to $L_{\phi}(E, T)$.

The Friedel-type oscillations in the LDOS at a straight step edge (Fig. 3(a)) are readily calculated in the following model. Let us consider a free noninteracting $2 \mathrm{D}$ electron gas with the dispersion being parabolic and isotropic in the center of the surface Brillouin zone (SBZ) $\left(k_{\|} \lesssim 0.2 \AA^{-1}\right)$ :
$E=E_{\Gamma}^{-}+\frac{\hbar^{2}}{2 m^{*}} k_{\|}^{2}$.

Here $E_{\Gamma}^{-}$is the surface-state band edge energy and the effective mass $m^{*}$ is positive for $\mathrm{s}-\mathrm{p}$ derived surface states on noble metals. By convention, the $x$ axis is chosen perpendicular to the step edge, i.e. $x$ denotes the distance from the step (Fig. 3(b)). Since we do not know anything about the step potential and to stay as general as possible, we model the step edge as a plane wave reflector with a coherent reflection amplitude $r\left(k_{x}\right)$ and a reflection phaseshift $\varphi\left(k_{x}\right)$, which both may depend on the energy which is in the electron motion perpendicular to the step. For coherent (elastic) processes the electron energy is conserved and since the straight step problem is invariant under translations along $y, k_{y}$ is conserved during the process, i.e. $k_{y}^{\prime}=k_{y}^{4}$. From energy and parallel momentum conservation it directly follows that $k_{x}^{\prime}=-k_{x}$. Thus the incoming plane wave

\footnotetext{
${ }^{4}$ We neglect the discrete nature of the translation symmetry and thus Bragg reflection [40], which is a good approximation since typical $\pi / k_{y}$ are much larger than next-nearest atom distances.
}

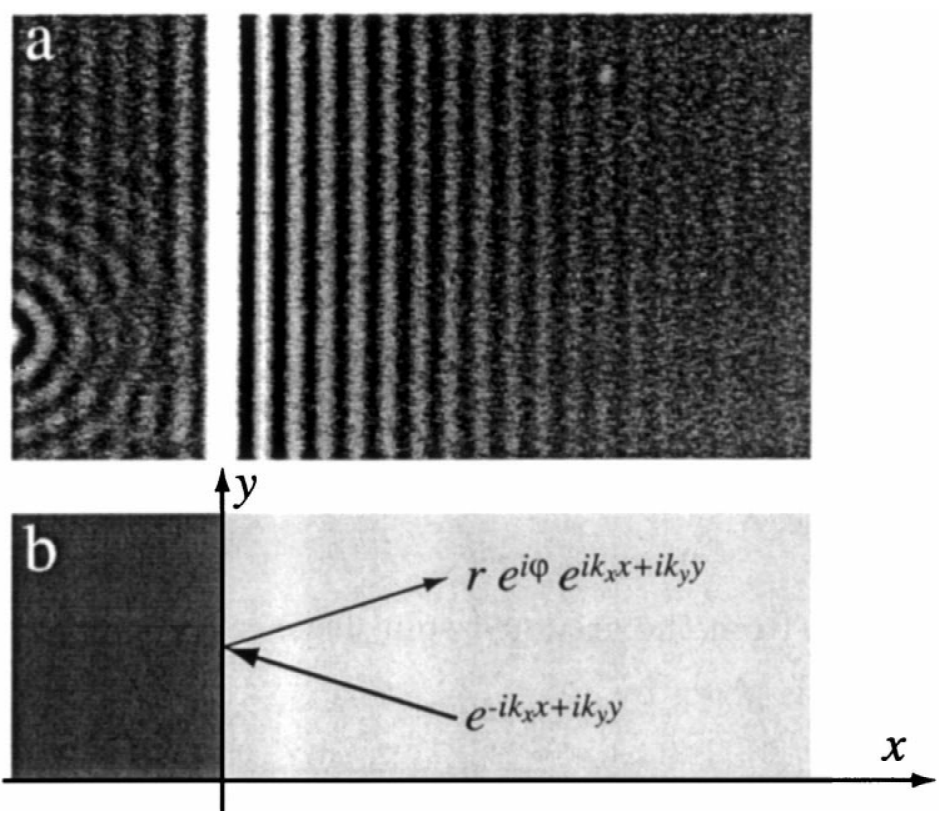

Fig. 3. (a) $247 \AA \times 138 \AA d I / d V$ image at a straight $\mathrm{Cu}(111)$ step edge. The step edge itself is imaged as a white stripe and the upper terrace is on the right hand side. To the very left a surface impurity is visible. LDOS oscillations at the step edge and impurity atom are clearly visible ( $T=4.9 \mathrm{~K}$, c.f., $\Delta V=101 \mathrm{mV}, \nu=5.7 \mathrm{kHz}$, stabilizing conditions: $V=600 \mathrm{mV}, I=3 \mathrm{nA}$ ). (b) Corresponding scattering schematics. 
$\mathrm{e}^{-i k_{x} x+i k_{y} y}$ has to be superimposed coherently by the reflected plane wave $r\left(k_{x}\right) \mathrm{e}^{i \varphi\left(k_{x}\right)} \mathrm{e}^{i k_{x} x+i k_{y} y}$, i.e. the wave function has the form

$$
\Psi_{E, k_{y}}(x, y)=\left(\mathrm{e}^{-i k_{x} x}+r\left(k_{x}\right) \mathrm{e}^{i \varphi\left(k_{x}\right)} \mathrm{e}^{i k_{x} x}\right) \mathrm{e}^{i k_{y} y}
$$

where $k_{x}=\sqrt{2 m^{*}\left(E-E_{\Gamma}^{-}\right) / \hbar^{2}-k_{y}^{2}}$. Electrons are not only reflected at the step edge but may be transmitted into the surface states on the adjacent terrace (with probability $t^{2}\left(k_{x}\right)$ ) or, since the 2D electron gas may be coupled to the bulk electrons at the step edge, they may be absorbed at the step (with probability $\left.a^{2}\left(k_{x}\right)\right)$. Absorption then means scattering from surface into bulk states. For simplicity we disregard the possible $k_{y}$ dependence of $a^{2}\left(k_{x}\right)$ and will reason later on that this does not change the final result for the LDOS at the step. Particle conservation implies $r^{2}\left(k_{x}\right)+t^{2}\left(k_{x}\right)+a^{2}\left(k_{x}\right)=1$. Since there is no net flux of electrons from surface states into bulk states, as much electrons must be emitted into surface states as are absorbed by bulk states, i.e. $a^{2}=e^{2}$, where $a^{2}$ and $e^{2}$ are the $k_{x}-k_{y}$-averaged probabilities for absorption and emission. We further assume that the probability distribution for emission is the very same as for absorption, implying in this case $a^{2}\left(k_{x}\right)=e^{2}\left(k_{x}\right)$ (also this does not alter the final result, as seen below). The number $N$ of electrons per unit area at location $(x, y)$ with energy less than $E$ is given by incoherent summation over $\left|\Psi_{E, k_{y}}(x, y)\right|^{2}$ of Eq. (6), the transmitted electrons from the left hand side and the emitted electrons:

$$
\begin{aligned}
N(E, x)= & 2 \int_{0}^{k_{\mathrm{E}}} \frac{\mathrm{d} k_{x}}{2 \pi} \int_{-\sqrt{k_{\mathrm{E}}^{2}-k_{x}^{2}}}^{\sqrt{k_{\mathrm{E}}^{2}-k_{x}^{2}}} \frac{\mathrm{d} k_{y}}{2 \pi}\left(\left(1+r^{2}\left(k_{x}\right)\right.\right. \\
& \left.+2 r\left(k_{x}\right) \cos \left(2 k_{x} x+\varphi\left(k_{x}\right)\right)\right)+t^{2}\left(k_{x}\right) \\
& \left.+e^{2}\left(k_{x}\right)\right) \\
= & \frac{2}{\pi} \int_{0}^{k_{\mathrm{E}}} \frac{\mathrm{d} k_{x}}{2 \pi}\left(2+2 r\left(k_{x}\right) \cos \left(2 k_{x} x\right.\right. \\
& \left.\left.+\varphi\left(k_{x}\right)\right)\right) \sqrt{k_{\mathrm{E}}^{2}-k_{x}^{2}} .
\end{aligned}
$$

Here the factor 2 comes from the assumed spin degeneracy and we have used $t^{2}\left(k_{x}\right)+e^{2}\left(k_{x}\right)=1-$ $r^{2}\left(k_{x}\right) . k_{\mathrm{E}}$ is given by
$k_{\mathrm{E}}=\sqrt{2 m^{*}\left(E-E_{\Gamma}^{-}\right) / \hbar^{2}}$.

The LDOS at the step edge is then readily calculated from Eq. (7):

$$
\begin{aligned}
\rho_{\text {step }}(E, x) & =\frac{\partial}{\partial E} N(E, x) \\
& =\frac{2}{\pi} L_{0} \int_{0}^{k_{\mathrm{E}}} d k_{x} \frac{1+r\left(k_{x}\right) \cos \left(2 k_{x} x+\varphi\left(k_{x}\right)\right)}{\sqrt{k_{\mathrm{E}}^{2}-k_{x}^{2}}} .
\end{aligned}
$$

Here $L_{0}$ is the DOS of a free electron gas, i.e. $L_{0}=m^{*} / \pi \hbar^{2}$. For $\operatorname{Ag}(111)$ the phaseshift $\varphi\left(k_{x}\right)$ has experimentally been shown to lie close to $-\pi$ independent of $k_{x}$ [41]. Furthermore, numerical integration of Eq. (9) showed, that the results for an arbitrary $\varphi\left(k_{x}\right)$ distinguishes itself from the result for $\varphi\left(k_{x}\right)=-\pi$ mainly by a mere $x$-translation of the order of $(\varphi+\pi) / k_{\mathrm{E}}$. For these two reasons we set $\varphi\left(k_{x}\right)=-\pi$ in the following. Under these conditions and with a reasonable $k_{x}$ dependence of $r\left(k_{x}\right)$ $[40,41]$ one finds

$\rho_{\text {step }}(E, x) \approx L_{0}\left(1-r\left(k_{\mathrm{E}}\right) J_{0}\left(2 k_{\mathrm{E}} x\right)\right)$,

where $J_{0}$ is the Bessel function of order zero. The relation is exact in the case of an energy independent reflection amplitude. The oscillations seen in Fig. 3 can thus be understood in terms of the oscillating part of Eq. (10), i.e. the $J_{0}\left(2 k_{\mathrm{E}} x\right)$ term. The asymptotic behavior of $J_{0}(u)$ is $\sqrt{2 / \pi u} \cos (u-\pi / 4)$, and thus, in a 2D electron gas, there is an intrinsic $1 / \sqrt{x}$ decay of the Friedel-type LDOS oscillations at a step edge $^{5}$. This decay for $\mathrm{D}>1$ comes from the fact that all $k_{x}$ values from 0 up to $k_{\mathrm{E}}$ contribute to the LDOS at fixed energy $E$. A decay of the interference patterns in Fig. 3 is clearly seen. But it is not a priori clear that this measured decay is governed by the intrinsic $1 / \sqrt{x}$ decay, since additional inelastic processes on the terrace may alter the decay behavior as discussed below. The LDOS in Eq. (10) asymptotically approaches the constant value $L_{0}$ far away from the step, justifying the assumptions made on $a^{2}\left(k_{x}\right)$ and $e^{2}\left(k_{x}\right)$.

Starting from $\rho_{\text {step }}$ in Eq. (10) the tunnel current

\footnotetext{
${ }^{5}$ An intrinsic decay of the LDOS oscillations is absent in 1D, but even more pronounced in $3 \mathrm{D}$.
} 
close to a step edge for bias voltages $e|V| \ll \bar{W}$ $\left(2 \bar{W}=W_{\mathrm{s}}+W_{\mathrm{t}}\right.$, where $W_{\mathrm{s}}$ and $W_{\mathrm{t}}$ are the work functions of sample and tip, respectively) is given by $[42,43]$

$$
\begin{aligned}
I(V, T, x, y, s) \propto & \mathrm{e}^{-2 s \sqrt{\frac{m_{e}}{\hbar^{2}}} \sqrt{2 \bar{W}}} \int_{-\infty}^{\infty} \mathrm{d} E \rho_{\mathrm{s}}(E, x, y) \\
& \times \rho_{\mathrm{t}}(E-e V) g(E, V, T),
\end{aligned}
$$

where $g(E, V, T)=f(E-e V, T)-f(E, T) ; f(E, T)$ is the Fermi-Dirac distribution function, $\rho_{\mathrm{s}}$ and $\rho_{\mathrm{t}}$ are the DOS of sample and tip, respectively. $s$ denotes the tip-sample separation which is related to the tip height $\left.z(x, y)\right|_{I, V}$, measured in constant current line scans, by

$\left.z(x, y)\right|_{I, V}=\left.s(x, y)\right|_{I, V}+t(x, y)$.

Here $t(x, y)$ characterizes the topography of the surface, i.e., a virtual plane passing through the surface atomic nuclei. In expression (11) for the tunneling current, $\rho_{\mathrm{s}}(E, x, y)$ has to be replaced by the sum of $\rho_{\text {step }}(E, x)$ and a constant bulk contribution $\rho_{\mathrm{b}}$. We assume a constant tip DOS in the narrow energy intervals of interest. The current can then be written

$I(V, T, x, s) \propto e^{-2 s \sqrt{\frac{m_{e}}{\hbar^{2}}}} \sqrt{2 \bar{W}}\left(\int_{-\infty}^{\infty} \mathrm{d} E\left(\rho_{\mathrm{b}}+\rho_{\text {step }}(E, x)\right) f(E-e V, T)\right.$

$\left.-\int_{-\infty}^{\infty} \mathrm{d} E\left(\rho_{\mathrm{b}}+\rho_{\text {step }}(E, x)\right) f(E, T)\right)$,

Following Adawi [44] the temperature effect due to the broadening of the Fermi function in the integrands of Eq. (13) can be approximated by performing the integration at $T=0 \mathrm{~K}$ and by multiplying the oscillating parts of the resulting functions with $\xi_{k_{\mathrm{E}}}$ / $\sinh \xi_{k_{\mathrm{E}}}$, where

$\xi_{k_{\mathrm{E}}}=x \frac{2 \pi m^{*}}{\hbar^{2}} \frac{k_{\mathrm{B}} T}{k_{\mathrm{E}}}$

and the energy $E$ for the evaluation of $k_{\mathrm{E}}$ has to be chosen at the cut-off of the Fermi function, i.e. $E_{\mathrm{F}}+e V$ and $E_{\mathrm{F}}$ for the first and second term in Eq. (13), respectively. The integrations at $T=0 \mathrm{~K}$ are readily evaluated and one obtains the following expression for the tunneling current close to a step edge

$$
\begin{aligned}
& I(V, T, x, s) \propto \mathrm{e}^{-2 s} \sqrt{\frac{m_{e}}{\hbar^{2}}} \sqrt{2 \bar{W}}\left(e V\left(L_{0}+\rho_{\mathrm{b}}\right)-r L_{0} \frac{\hbar^{2}}{2 m^{*}} \frac{1}{x}\right. \\
& \left.\quad \times\left(\frac{\xi_{k_{\mathrm{eV}}}}{\sinh \xi_{k_{\mathrm{eV}}}} k_{\mathrm{eV}} J_{1}\left(2 k_{\mathrm{eV}} x\right)-\frac{\xi_{k_{\mathrm{F}}}}{\sinh \xi_{k_{\mathrm{F}}}} k_{\mathrm{F}} J_{1}\left(2 k_{\mathrm{F}} x\right)\right)\right) .
\end{aligned}
$$

Here $J_{1}$ is the first order Bessel function. We have assumed that the step reflection amplitude $r$ is the same for all $k_{x}$ wave vectors contributing to $I(V, T, x, s)$, which is a good approximation when $e|V| \ll\left|E_{\Gamma}^{-}\right|$. For low bias voltages the effective reflection amplitude $r$ is given by $r\left(k_{\mathrm{F}}\right)$. Numerical solution of the integral in Eq. (11) with $\rho_{\text {step }}$ from Eq. (10) shows that the Adawi approximation in Eq. (15) is very accurate. By inverting Eq. (15) the constant-current tip-sample distance at a step edge is obtained:

$$
\begin{aligned}
\left.s(x)\right|_{I, V}= & \frac{1}{2 \sqrt{\frac{m_{e}}{\hbar^{2}}} \sqrt{2 \bar{W}}} \ln \left(1-r \frac{1}{e V} \frac{L_{0}}{\rho_{\mathrm{b}}+L_{0}} \frac{\hbar^{2}}{2 m^{*}} \frac{1}{x}\right. \\
& \left.\times\left(\frac{\xi_{k_{\mathrm{eV}}}}{\sinh \xi_{k_{\mathrm{eV}}}} k_{\mathrm{eV}} J_{1}\left(2 k_{\mathrm{eV}} x\right)-\frac{\xi_{k_{\mathrm{F}}}}{\sinh \xi_{k_{\mathrm{F}}}} k_{\mathrm{F}} J_{1}\left(2 k_{\mathrm{F}} x\right)\right)\right)
\end{aligned}
$$

Since for $u \gg 1 J_{1}(u)$ behaves as $\sqrt{2 / \pi u} \cos (u-$ $3 \pi / 4), s(x)$ asymptotically falls off like $1 / x^{3 / 2}$ at zero temperature, i.e. faster than the corresponding LDOS. This is due to the wave vector spread in the integral over the LDOS. It is seen in Eq. (15) that the amplitude of the oscillations scales with $k_{\mathrm{eV}} / \mathrm{eV}$, meaning that the higher the tunneling bias, the smaller is the amplitude of the interference patterns. Furthermore, the wave vector spread leads to a damping of the interference patterns which is more pronounced for larger bias values (see Section 3). Altogether, the effect of the LDOS oscillations on $\left.s(x)\right|_{I, V}$ is more pronounced at low bias values and is almost not visible at higher biases, where the wave vector spread washes out the interference effects in $\left.s(x)\right|_{I, V}$.

To demonstrate the validity of our $\left.s(x)\right|_{I, V}$ model we present in Fig. 4(a) the fit by Eq. (16) to a topographic linescan taken perpendicularly to a monoatomic $\operatorname{Ag}(111)$ step. For $\operatorname{Ag}(111)$ the electron wave vectors $k_{\mathrm{eV}}, k_{\mathrm{F}}$ and the effective mass $m^{*}$ are 


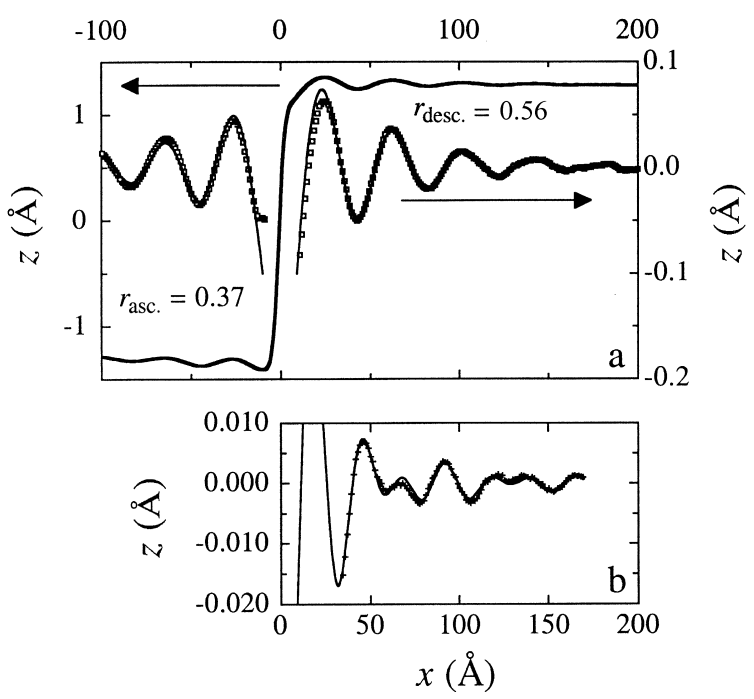

Fig. 4. Constant-current linescan taken across a $\operatorname{Ag}(111)$ step (a) at $V=10 \mathrm{mV}$ and $T=77.3 \mathrm{~K}$, (b) at $V=100 \mathrm{mV}$ and $T=3.5 \mathrm{~K}$ $(I=1.0 \mathrm{nA}$ in (a) and (b)). The solid lines are fits using Eq. (16) (see text). The agreement between experiment and model is striking; also pronounced beating effects appearing at larger bias voltage as in (b) are perfectly reproduced. The only free fit parameters in (a) are the two reflection amplitudes $r_{\text {desc }}$ and $r_{\text {asc }}$ for descending and ascending steps.

known from the dispersion $\left(E_{0}^{2 D}=-65 \mathrm{meV}, m^{*}=\right.$ $0.40 m_{e}$, see $\left.[18,45]\right)$. The ratio $L_{0} /\left(\rho_{\mathrm{b}}+L_{0}\right)=0.64$ has been estimated from $\mathrm{d} I / \mathrm{d} V$ data on a clean terrace [45]. Furthermore, by ramping $z$ and measuring the tunneling current $I$ we have determined the apparent barrier height $\bar{W}=(3.1 \pm 0.1) \mathrm{eV}$ for $\mathrm{Ag}(111)$ [45]. Except from the reflection amplitudes $r_{\text {desc }}$ and $r_{\text {asc }}$ for the descending and ascending side of the step, respectively, all parameters entering Eq. (16) are thus known. The good agreement between model and experiment is evident. From our fit we obtain quite different reflection amplitudes $r$ on the upper and on the lower terrace. For electrons being reflected by the ascending step $r_{\text {asc }}$ is $1.8 \pm 0.4$ times smaller than for those approaching a descending step. These $r$-values represent the reflection amplitude at the Fermi level, $r\left(k_{\mathrm{F}}\right)$, since the linescan has been taken at low bias. The linescan in Fig. 4(b) has been performed at a bias voltage of $100 \mathrm{mV}$. It clearly shows the beating of the Bessel functions at $k_{100 \mathrm{meV}}$ and $k_{\mathrm{F}}$ (see Eq. (16)). The oscillations and beating in $z(x)$ down to amplitudes of $1 / 1000 \AA$ are perfectly described by Eq. (16). Note the difference in amplitude of the oscillations in Fig. 4(a) and (b). At $10 \mathrm{mV}$ the amplitude of the oscillations are almost a factor of 10 larger than at $100 \mathrm{mV}$, confirming that the wave vector spread washes out the coherence effects.

So far we included only possible loss of coherence during scattering at the step edge itself (via $\left.r\left(k_{\mathrm{E}}\right)\right)$ in our model for the LDOS at step edges (Eq. (9)), but completely disregarded inelastic processes on the terrace. The latter processes include e-e and e-ph scattering. Not including them in the model was justified, since the measurements in Fig. 4 have been performed at low temperature and low energies $(E-$ $E_{\mathrm{F}}<0.5 \mathrm{eV}$ ), where $L_{\mathrm{e}-\mathrm{e}}$ and $L_{\mathrm{e}-\mathrm{ph}}$ are much larger than the intrinsic LDOS decay length (see below for estimates of the order of $L_{\phi}$ in this regime). Fig. 5 illustrates electron scattering processes at step edges without and with inelastic processes on the terrace. In the absence of inelastic processes on the terrace an electron wave packet of wave vector $\left(-k_{x}, k_{y}\right)$ will be reflected partially by the step edge into a state characterized by $\left(k_{x}, k_{y}\right)$ and will interfere coherently with the incoming wave packet, leading to the well known interference patterns. At the step edge itself we allowed for inelastic processes which further reduce the step reflection amplitude $r\left(k_{x}\right)$. Since these processes are located at the step edge, an electron starting at distance $x$ from the step will

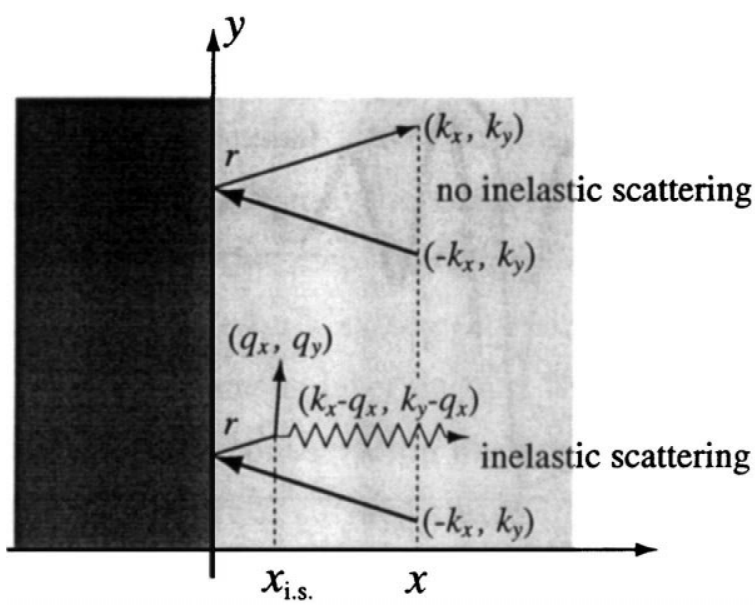

Fig. 5. Schematics of electron reflection at a straight step edge, without (top) and with inelastic scattering (bottom) at the location $x_{\text {i.s. }}$. Possible inelastic processes include e-ph (as sketched) and $\mathrm{e}-\mathrm{e}$ scattering. 
come back to this distance with a probability that is independent of $x$. Thus, inelastic processes at steps lead to an overall reduction of the LDOS oscillation amplitude, but not to damping (Eq. (10)). If we take inelastic processes on the terrace into account, then the electron may be scattered out of its state into another quantum state $\left(q_{x}, q_{y}\right)$ somewhere on its way from distance $x$ to the step or from the step to distance $x$ again. Since this is an inelastic process, the energy of state $\left(q_{x}, q_{y}\right)$ is different from the energy of $\left(k_{x}, k_{y}\right)$, e.g. the process involves absorption or emission of a phonon. If we assume that the system is homogeneous, then an inelastic process occurs with a constant probability $\mathrm{d} \ell / L_{\phi}$ per length unit $\mathrm{d} \ell$, i.e. the probability that the electron is in the same quantum state after a distance $\ell$ is $\mathrm{e}^{-\ell / L_{\phi}}$. The distance an electron wave packet in state $\left(-k_{x}, k_{y}\right)$ has to travel starting from distance $x$, going to the step and then back to distance $x$, where it can interfere with the incoming $\left(-k_{x}, k_{y}\right)$, is given by $2 x k_{\mathrm{E}} / k_{x}$, where $k_{\mathrm{E}}^{2}=k_{x}^{2}+k_{y}^{2}$. The probability that the electron in state $\left(-k_{x}, k_{y}\right)$ gets back to distance $x$ in state $\left(k_{x}, k_{y}\right)$ is reduced by a factor $\exp \left(-2\left(k_{\mathrm{E}} x\right)\right.$ $\left.k_{x} L_{\phi}\right)$, and therefore, under inclusion of inelastic processes, the LDOS of the $2 \mathrm{D}$ electron gas at a step edge in Eq. (9) reads:

$\rho_{\text {step }}(E, x)=\frac{2}{\pi} L_{0} \int_{0}^{k_{\mathrm{E}}} d k_{x} \frac{1-r\left(k_{x}\right) \mathrm{e}^{-2 \frac{k_{\mathrm{E}} x}{k_{x} L_{\phi}}} \cos \left(2 k_{x} x\right)}{\sqrt{k_{\mathrm{E}}^{2}-k_{x}^{2}}}$.

Again, numerical integration of Eq. (17) shows that for $x>\pi / k_{\mathrm{E}}$ and a reasonable $k_{x}$ dependence of $r\left(k_{x}\right)$ $[40,41], \rho_{\text {step }}(E, x)$ can very well be approximated by

$\rho_{\text {step }}(E, x) \approx L_{0}\left(1-r\left(k_{\mathrm{E}}\right) \mathrm{e}^{-2 \frac{x}{L_{\phi}}} J_{0}\left(2 k_{\mathrm{E}} x\right)\right)$.

As seen in Eq. (18) inelastic processes on the terrace lead to an additional damping of the LDOS interference patterns. By quantitatively studying the decay of these interference patterns at straight step edges with STM, one can investigate inelastic processes like e-e and e-ph scattering. We emphasize that $L_{\phi}$ as defined here (and elsewhere [46]) does not account for coherence loss at scattering centers themselves. Hence, our measured lifetime directly reflects the lifetime of surface-state electrons on an ideal surface free of any defects.

Theoretical lifetimes usually refer to one single quasiparticle added to the Fermi sphere (ground state) [3]. Photoelectron spectroscopic methods are far from this idealized situation since many quasiparticles are usually excited with the photon (electron) pulse, leading to a highly non-equilibrium quasiparticle distribution. STM, on the contrary, comes close to the theoretical scenario. To illustrate this statement let us use the picture put forward by Heller et al. [47]: at positive bias voltages (similar arguments can be made for negative bias) electrons tunnel from the tip to the sample surface. On the sample this electron wave travels away from the tip. If it encounters scattering centers like steps or impurities, it may be scattered and return to the tip, where it will interfere constructively or destructively with the amplitude leaving the tip. The electron can be injected at a well defined energy $\mathrm{eV}$ above the Fermi surface by choosing the appropriate bias voltage $V$. In this picture it gets clear, that with the STM tip one injects electrons, whose properties can be probed by the very same tip. Since at typical tunneling currents of $1 \mathrm{nA}$ an electron is injected about every $0.16 \mathrm{~ns}$ and since typical lifetimes of these surface-state electrons are in the fs range (see below), only one single quasiparticle is probed at a time. STM therefore offers in principle the experimental realization of the simple picture used in theory, where one single quasiparticle is added to the Fermi sphere.

\section{Electron-electron interaction}

To learn about $\mathrm{e}-\mathrm{e}$ interaction of $\mathrm{s}-\mathrm{p}$ derived surface-state electrons on noble metals we have studied the decay of quantum interference patterns at step edges as a function of the quasiparticle excess energy (Fig. 1). With a simple model we have been able to extract $L_{\phi}(E)$ from $\mathrm{d} I / \mathrm{d} V$ scans acquired under closed feedback loop conditions at step edges for the Shockley type surface states on $\operatorname{Ag}(111)$ and $\mathrm{Cu}(111)$.

Fig. 6 shows a constant-current image of a $\mathrm{Cu}(111)$ step edge at $V=1.4 \mathrm{~V}$ in (a) and the closed feedback $\mathrm{d} I / \mathrm{d} V$ image taken simultaneously in (b). 

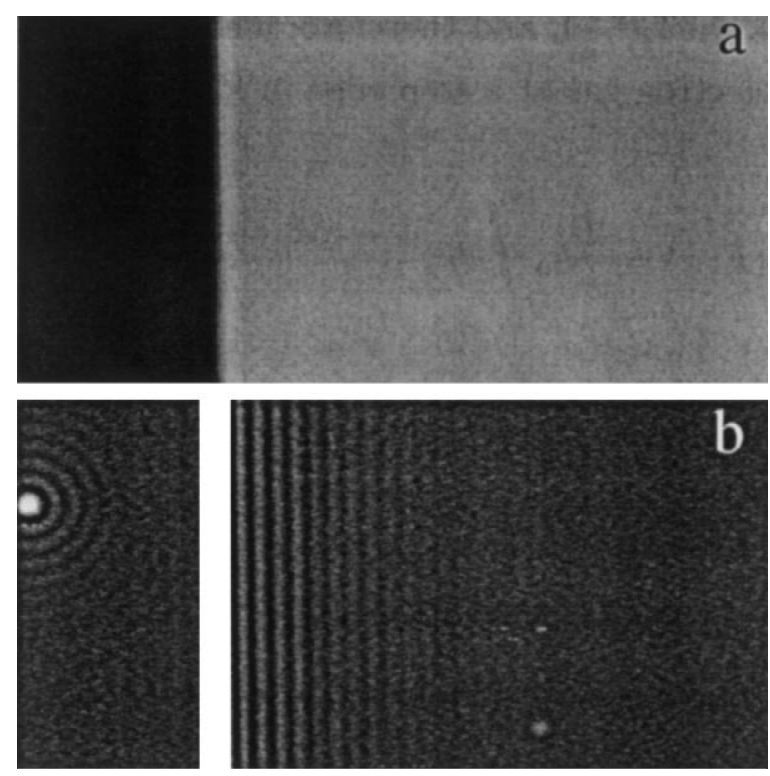

Fig. 6. (a) Constant-current image of a $\mathrm{Cu}(111)$ step edge: 280 $\AA \times 138 \AA, V=1.4 \mathrm{~V}, I=7 \mathrm{nA}$. (b) $\mathrm{d} I / \mathrm{d} V$ image taken simultaneously with (a). Standing wave patterns at static scatterers as steps and impurities are clearly visible $(T=4.9 \mathrm{~K}$, c.f., $\Delta V=135$ $\mathrm{mV}, \nu=5.72 \mathrm{kHz}$ ).

Again, Friedel-type oscillations in the LDOS are responsible for the clearly visible spatial oscillations in Fig. 6(b). For our experiment we have chosen straight step edges with a defect free area larger than $250 \AA \times 250 \AA$ on the adjacent upper terrace (Fig. 6 ). By doing so we are sure that the local elastic mean free path $L_{\mathrm{m}}$ is considerably larger than the measured $L_{\phi}$ [1], and thus the LDOS oscillations at the step are not influenced by other static scattering centers. In order to evaluate the decay of the standing waves at straight step edges as shown in Fig. 6 the $d I / d V$ images have been slightly rotated to align the step edge vertically, and then we have averaged the $\mathrm{d} I / \mathrm{d} V$ data over several line scans. Typical averaged $\mathrm{d} I / \mathrm{d} V$ data are presented in Fig. 7(a). To interpret this data we start with the general expression for the tunneling current $I[42,43,48]$

$$
\begin{aligned}
I(V, T, x, y, s) & \propto \int_{-\infty}^{\infty} \mathrm{d} E \rho_{\mathrm{s}}(E, x, y) \rho_{\mathrm{t}}(E-e V) \\
& \times \mathscr{T}(E, V, s)[f(E-e V, T)-f(E, T)],
\end{aligned}
$$

where $\rho_{\mathrm{t}}$ is the DOS of the tip, $x$ and $y$ characterize

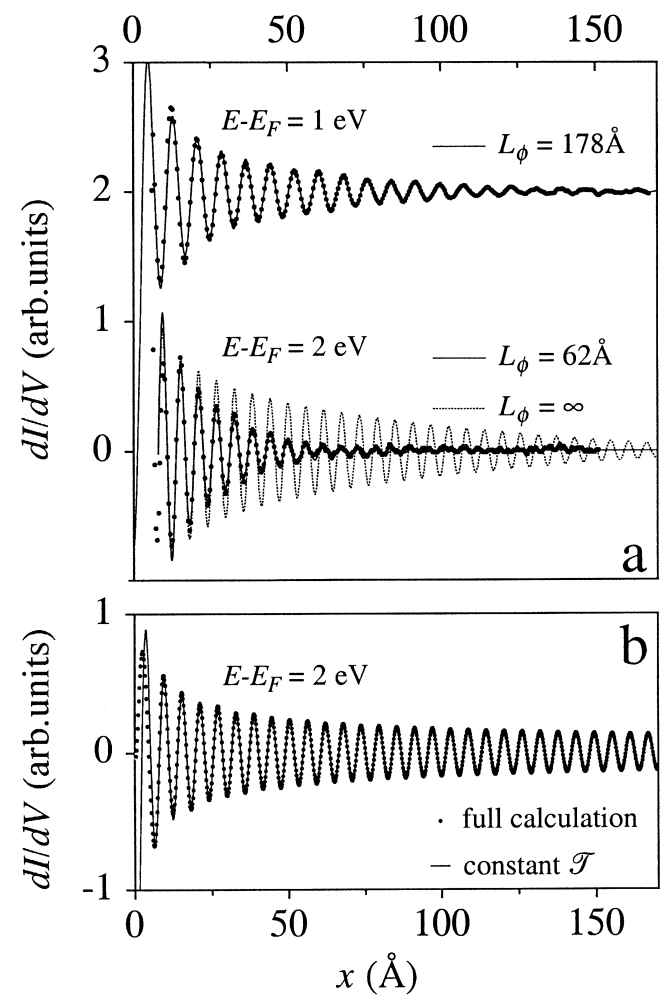

Fig. 7. (a) Typical $\mathrm{d} I / \mathrm{d} V$ data perpendicular to a descending $\mathrm{Cu}(111)$ step obtained by averaging over several line scans of a $\mathrm{d} I / \mathrm{d} V$ image as shown in Fig. 6(b). The data at $1 \mathrm{eV}$ and $2 \mathrm{eV}$ were taken with a stabilizing current of $5 \mathrm{nA}$ and $10 \mathrm{nA}$ and a $\Delta V$ of $119 \mathrm{mV}$ and $156 \mathrm{mV}$, respectively. The solid lines depict the fits with Eqs. (21) and (22). The significance of the deduced $L_{\phi}$ is demonstrated by the dashed line: neglecting inelastic processes by setting $L_{\phi}=\infty$ leads to a much slower decay rate than observed $(T=4.9 \mathrm{~K}$, c.f., $\nu=5.72 \mathrm{kHz}$ ). (b) Comparison between the full calculation of $\mathrm{d} I / \mathrm{d} V$ with Eqs. (19), (18) and (20) and the result obtained by setting $\mathscr{T}$ constant $\left(T \rightarrow 0, L_{\phi} \rightarrow \infty\right.$, typical $\mathrm{Cu}(111)$ parameters: $\left.W_{\mathrm{s}}=W_{\mathrm{t}}=4.5 \mathrm{eV}, r=0.5[40]\right)$.

the lateral position on the sample and $s$ the distance between tip and sample measured from a virtual plane passing through the uppermost atoms. $\rho_{\mathrm{s}}$ is the LDOS of the sample in this virtual plane. The tip LDOS $\rho_{\mathrm{t}}$ is assumed to be constant which is justified since we are only interested in lateral variations of $\mathrm{d} I / \mathrm{d} V$. We use the transmission factor [49]

$\mathscr{T}(E, V, s)$

$=\mathrm{e}^{-s \sqrt{2 m_{e} / \hbar^{2}}\left(\sqrt{W_{\mathrm{t}}-E+e V}+\sqrt{-E\left(1-m * / m_{e}\right)-m * / m_{e} E_{\Gamma}^{-}+W_{\mathrm{s}}}\right)}$, 
which accounts for the $p_{\|}$dependence of the vacuum barrier penetration of surface-state wave functions ${ }^{6}$. The work function of the sample, $W_{\mathrm{s}}$, can be considered as a constant for our purposes since we have found its reduction at steps due to the Smoluchowski effect [50] to be localized to $\pm 3 \AA$ around the step edge. For $\rho_{\mathrm{s}}(E, x)$ in Eq. (19) we use $\rho_{\mathrm{b}}+\rho_{\text {step }}(E, x)$ from Eq. (18), appropriate for a straight step edge in presence of inelastic processes. Since we are only interested in spatial variations of $\mathrm{d} I / \mathrm{d} V$ the bulk contribution to the surface DOS, $\rho_{\mathrm{b}}$, is assumed to be constant.

In the following we prove that $\mathscr{T}$ can be assumed constant for our purposes due to the facts, that firstly, at relatively large bias voltages the constant-current tip sample distance $\left.s(x)\right|_{I, V}$ is not influenced by the LDOS oscillations, and secondly, the energy dependence of $\mathscr{T}(E, V, s)$ can be neglected since the energies entering in $\mathrm{d} I / \mathrm{d} V$ all lie in the window of the lock-in bias modulation $e \Delta V$ which is much smaller than $\bar{W}$. With Eqs. (19), (18) and (20) we have calculated $\mathrm{d} I / \mathrm{d} V$ numerically, using the constant-current tip sample distance $5 \AA+\left.s(x)\right|_{I, V}$ of Eq. (16), and typical $5 \mathrm{~K}$ parameters for the $\mathrm{Cu}(111)$ and $\operatorname{Ag}(111)$ surface states $\left(E_{0}^{2 D}=-65 \mathrm{meV}, m^{*}=\right.$ $0.40 m_{e}$ for $\operatorname{Ag}(111)$ and $E_{0}^{2 D}=-420 \mathrm{meV}, m^{*}=$ $0.40 m_{e}$ for $\mathrm{Cu}(111)$, see $\left.[7,17,18,29,45]\right)$. By doing so we simulate the $\mathrm{d} I / \mathrm{d} V$ imaging mode under closed feedback loop conditions. The result of such a calculation is depicted in Fig. 7(b) (dots) and compared with the result obtained by setting the transmission factor $\mathscr{T}$ constant (full line). From plots as shown in Fig. 7(b) it is clear that the energy and gap width dependence of the transmission factor of Eq. (20) is neither responsible for a faster decay of the oscillations in $\mathrm{d} I / \mathrm{d} V$ at steps nor does it change the oscillation period and phase (at least not in the bias regime of $0.3 \mathrm{~V}-3.5 \mathrm{~V}$ ). For the sake of a faster fit procedure we thus can safely set the transmission factor $\mathscr{T}=$ const, which is an excellent approximation for $x>\pi / k_{\mathrm{E}}$ and $0.3 \mathrm{~V}<V<3.5 \mathrm{~V}$. Under these circumstances the integral in Eq. (19) with $\rho_{\mathrm{b}}+\rho_{\text {step }}(E, x)$ from Eq. (18) can be analytically

\footnotetext{
${ }^{6}$ Since these states are $2 \mathrm{D}, p_{\|}$is completely characterized by $E$ and it enters implicitly in $\mathscr{T}$ via $E$.
}

solved, and the laterally varying part of the current, $I^{\sim}$, is given by (see Eq. (15)):

$$
\begin{aligned}
& I^{\sim}(V, T, x) \propto-\frac{1}{x} \mathrm{e}^{-2\left(x / L_{\phi}\right)} \\
& \quad \times\left(\frac{\xi_{k_{\mathrm{eV}}}}{\sinh \xi_{k_{\mathrm{eV}}}} k_{\mathrm{eV}} J_{1}\left(2 k_{\mathrm{eV}} x\right)-\frac{\xi_{k_{\mathrm{F}}}}{\sinh \xi_{k_{\mathrm{F}}}} k_{\mathrm{F}} J_{1}\left(2 k_{\mathrm{F}} x\right)\right) .
\end{aligned}
$$

Please note that for lower bias values the assumption of a constant transmission factor is not correct and the closed feedback $\mathrm{d} I /\left.\mathrm{d} V(x)\right|_{\mathrm{V}}$ can not directly be interpreted in terms of the LDOS [51]. An open feedback loop approach has to be chosen to measure $\rho_{\mathrm{s}}$ for low biases $[30,45,49]$. Since we have measured our $\mathrm{d} I / \mathrm{d} V$ data with a non-negligible lock-in bias modulation $\Delta V$ (peak-to-peak) we do not fit our data with the analytical derivative of Eq. (21) but with its lock-in derivative given by

$\mathrm{d} I / \mathrm{d} V(V, \Delta V) \propto \int_{0}^{2 \pi} \sin t \cdot I\left(V+\frac{\Delta V}{2} \sin t\right) \mathrm{d} t$,

where Eq. (21) has to be inserted for the current $I$. Note that in the limit of $\Delta V \rightarrow 0$ the lock-in output of Eq. (22) coincides with the real derivative. By using Eqs. (21) and (22) to fit our data we take fully account of modulation and temperature effects. The bias modulation actually leads to an apparent decay in $d I / d V$ beyond the one present in the LDOS pattern. The decay length $L_{\Delta V}$ of this additional $\mathrm{d} I / \mathrm{d} V$ decay is of the order of

$L_{\Delta V} \sim \frac{\hbar^{2} k_{\mathrm{eV}}}{m^{*} e \Delta V}$.

(The energy spread of $e \Delta V$ leads to a corresponding wave vector spread $\Delta k$ which then leads to a decay on a length scale of $1 / \Delta k$ ). $L_{\Delta V}$ can be considerable in our experiments and it is therefore of great importance that we account for this effect with Eq. (22). If we just evaluated the apparent decay in $d I / d V, L_{\mathrm{dI} / \mathrm{dV}}$, we would underestimate the real phase-relaxation length, since $L_{\mathrm{d} / / \mathrm{d} V}^{-1}=L_{\phi}^{-1}+L_{\Delta V}^{-1}$.

Using Eqs. (21) and (22) to fit our $\mathrm{d} I / \mathrm{d} V$ line scans, we are left with four fit parameters: $L_{\phi}, k_{\mathrm{eV}}$, the step edge location and an overall proportionality factor. Remember that the latter fully accounts for 
loss of coherence during the scattering process at the step edge itself $\left(r\left(k_{x}\right)\right.$ in Eq. (17)) whereas the decay $L_{\phi}$ is only influenced by inelastic processes on the terrace, e.g. e-e or e-ph interaction. Fits to measured $\mathrm{d} I / \mathrm{d} V$ data for $\mathrm{Cu}(111)$ are depicted in Fig. 7(a) for two different bias values (i.e. injection energies). The fit range has been limited to $x>3 \pi /$ $2 k$ to ensure the validity of our approximations. The agreement between fits and data is excellent and the relevance of $L_{\phi}$ is demonstrated by plotting the calculated $\mathrm{d} I / \mathrm{d} V$ oscillations for $L_{\phi}=\infty$. By fitting $\mathrm{d} I / \mathrm{d} V$ data taken at different bias voltages $V$ (i.e. energies $\mathrm{eV}$ ) for $\mathrm{Ag}(111)$ and $\mathrm{Cu}(111)$ we obtain the dispersion relation $k_{E=\mathrm{eV}}$ [45,52], and the energy dependent phase-relaxation length $L_{\phi}(E)$ for $\mathrm{Cu}(111)$ and $\operatorname{Ag}(111)$, respectively.

To compare our results with theory, APS, and 2PPE measurements we have converted the measured $L_{\phi}$ into lifetimes $\tau_{\phi}$ via $\tau_{\phi}=L_{\phi} / v$, where $v$ is the group velocity of the quasiparticles at the particular energy, $v=\hbar k_{\mathrm{eV}} / m^{*}$. This conversion is correct since locally $L_{\phi} \ll L_{m}$ in our case [1]. The measured $\tau_{\phi}(E)$ values are shown in Fig. 8 for $\mathrm{Cu}(111)$ and $\operatorname{Ag}(111)$. The points in Fig. 8 have been determined by averaging over data sets obtained with different tips, at different step edges and different fit ranges. The fit range and bias modulation dependence of our $\tau_{\phi}$ data is minor, which confirms the validity of our model. The error bars are due to a slight tip dependence of our measurement and a 5\% uncertainty in the STM piezo calibration. Actually, the absolute values of the lifetimes have been found to depend slightly on the tip, whereas the energy dependence of $\tau_{\phi}$ is unaffected. This might be explained by the fact that tips are not radially symmetric and thus may collect surface-state electrons having different in-plane incidence angles $\alpha=$ $\arccos \left(k_{x} / k_{\mathrm{eV}}\right)$ with different probabilities. Thus, the integrand in Eq. (17) would have to be multiplied with a probability function $f\left(k_{x} / k_{\mathrm{eV}}\right)$. It turns out that a monotonically increasing (decreasing) $f\left(k_{x} / k_{\mathrm{eV}}\right)$ leads to a slower (faster) decay of $\rho_{\text {step }}$. We point out that the absence of an influence of the tunneling impedance on our measurement has been carefully checked by measuring $L_{\phi}$ at fixed bias and a stabilizing current that has been varied by two orders of magnitude around the usual values. Thus, we believe that the presence of the tip, i.e. the tip

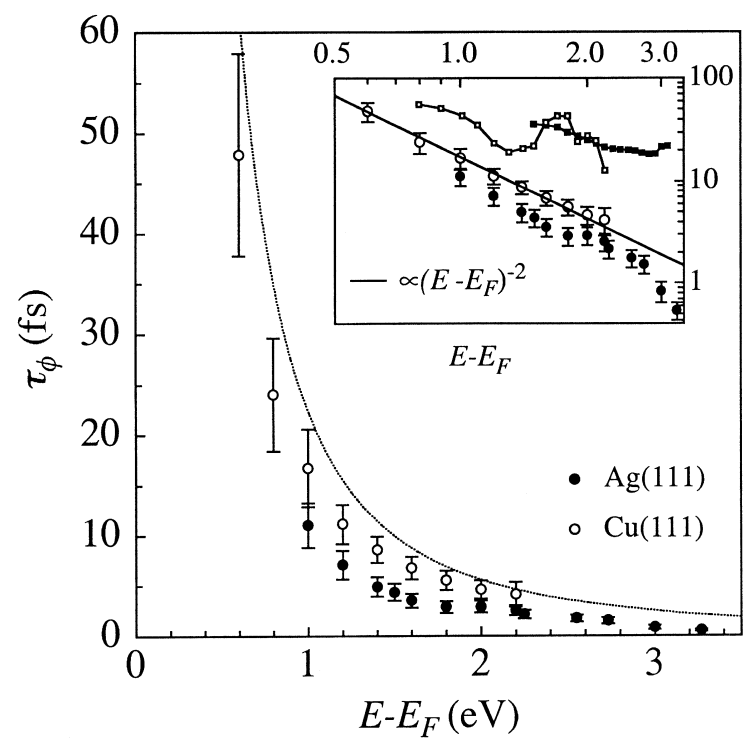

Fig. 8. Lifetimes of $\mathrm{s}-\mathrm{p}$ surface-state electrons as a function of excess energy determined as described in the text $(T=4.9 \mathrm{~K})$. The dotted line depicts the lifetimes predicted by 3D FLT for $\mathrm{Cu}$ (Eq. (1)): $\tau_{\mathrm{FLT}}=22.4 \mathrm{fs}^{2}\left(E-E_{\mathrm{F}}\right)^{-2}$. The inset shows the same data on a double logarithmic scale. The best inverse quadratic fit to the $\mathrm{Cu}$ data (full line) yields $\tau=17.1 \mathrm{fs}^{2}\left(E-E_{\mathrm{F}}\right)^{-2}$. The filled and open squares in the inset depict 2PPE data of $\mathrm{Cu}(111)$ bulk electron lifetimes measured by Ogawa et al. [27] and Knoesel et al. [28], respectively.

induced electric field or tip-surface interactions, does not influence the measured decay lengths.

Note that with our technique we avoid depopulation and cascade effects present in 2PPE and we probe only one excited electron at a time. Since electron-phonon lifetimes (typically 70 fs at $5 \mathrm{~K}$ ) are essentially independent of the quasiparticle energy for the energies of interest and exceed our measured lifetimes considerably (Fig. 1), we attribute the inelastic quasiparticle scattering rate $\tau_{\phi}^{-1}$ to e-e interaction, e.g. electron-hole pair creation ${ }^{7}$ Although surface-state electrons are bound to two dimensions they coexist with the underlying bulk electrons, and this opens up fully 3D decay channels, e.g. the e-e interaction is not restricted to the $2 \mathrm{D}$

\footnotetext{
${ }^{7}$ The energies of interest are well below the threshold for plasmon creation in $\mathrm{Ag}$ and $\mathrm{Cu}[53,54]$.
} 
electron gas itself but may have contributions from the bulk electrons. Surface-state electrons are efficiently screened by underlying bulk electrons, and one therefore expects that bulk electrons contribute to the e-e interaction of hot $s-p$ surface-state quasiparticles with the Fermi sphere. As can be seen in Fig. 8 our results for the surface-state lifetimes lie close to the $\tau_{0}\left(E-E_{\mathrm{F}}\right)^{-2}$ law predicted by 3D FLT for electron-hole pair creation (Eq. (1)): fits to our data yield $\tau_{0}^{\mathrm{Ag}}=10.4 \mathrm{fs}^{2} \mathrm{~V}^{2}$ and $\tau_{0}^{\mathrm{Cu}}=17.1 \mathrm{fs}^{2}$,

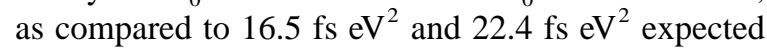
from FLT with $\mathrm{Ag}$ and $\mathrm{Cu}$ bulk parameters, respectively (see Fig. 8). Our surface-state lifetimes in Fig. 8 are slightly (but significantly) smaller than the lifetimes predicted in FLT for corresponding bulk electrons. Comparison of our data with more realistic models including the real band structure and exchange [55], which, for noble metals, predict larger lifetimes than $\mathrm{FLT}^{8}$, leads to an even larger deviation of our data from theory. In addition, recent 2PPE experiments confirm this trend towards larger bulk electron lifetimes for $\mathrm{Cu}(111)$ (See filled and open squares in the inset of Fig. 8. Note that these 2PPE data are depopulation lifetimes whereas we measure dephasing lifetimes. Depopulation and decoherence lifetimes could be discerned in interferometric 2PPE [8]). Therefore, we can state that $s-p$ surface-state electron lifetimes on noble metals are reduced with respect to bulk electron lifetimes. Calculations performed by Echenique et al. confirm our results [56]. Further theoretical modeling will be helpful to interpret our results in detail. Especially, the deviations of the $\tau_{\phi}$ data of $\operatorname{Ag}(111)$ above $2 \mathrm{eV}$ from the quadratic behavior of FLT should be related to the real band structure including $d$ bands.

To conclude this section, we would like to emphasize the possibility of studying also bulk quasiparticle lifetimes with STM, much in the way described here, since bulk electrons create as well

\footnotetext{
${ }^{8}$ Including exchange terms leads to a larger mean distance between electrons and thus an enhancement of e-e lifetimes. Including $d$ bands (i.e. the real band structure) introduces additional screening which increases the lifetimes as well.
}

standing wave patterns at structural defects on and below surfaces $[57,58]$.

\section{Electron-phonon interaction}

The general aim of this Section is to fully understand and model the thermal damping of interference patterns in 2D free electron gases. We present temperature dependent low-bias constant-current and $d I / d V$ measurements for $\mathrm{s}-\mathrm{p}$ derived surface-state electrons on noble metals in the temperature range $3.5 \mathrm{~K}$ to $178 \mathrm{~K}$. Although temperature dependent damping has been discussed earlier [30,33], we consider this Section valuable since it is more quantitative than the earlier studies. In particular, we have performed these quantitative temperature dependent decay studies to learn about electronphonon processes in noble-metal surface states, which dominate the electron decay rate at low excitation energy (Fig. 1).

Our investigation of the temperature induced spatial damping of standing waves is mostly based on constant-current line scans $\left.z(x)\right|_{I, V}$ taken perpendicularly to straight steps at low bias voltages. Such topographic data in the vicinity of a step are represented in Fig. 4. Although they are less directly related to the LDOS than $\mathrm{d} I / \mathrm{d} V$-profiles used to investigate e-e interaction in Section 3 (e.g. Fig. 7), higher resolution can generally be obtained in topographic data. Since e-ph damping involves much larger $L_{\phi}$ values compared to e-e damping of electrons at large bias voltages $V>1 \mathrm{~V}$ (Fig. 1), we need a better resolution here than the resolution that was necessary to learn about $\mathrm{e}-\mathrm{e}$ processes in Section 3. Experimental results presented in this Section have been obtained by averaging over several line scans which were recorded on the same surface spot, i.e. without $y$-displacement of the tip while scanning in $x$-direction. Note the resolution of $\approx 1 / 1000 \AA$ of such $z(x)$-data (Fig. 4(b)).

To interpret our constant-current line scans we include inelastic scattering processes in the formalism leading to Eq. (16). Introducing $\rho_{\text {step }}(E, x)$ from Eq. (18) into Eq. (13) and going through the calculation sketched in Section 2 leads to the following expression for the constant-current tip-sample 
distance at a straight step edge in presence of inelastic processes:

$$
\begin{aligned}
& \left.s(x)\right|_{I, V}=\frac{1}{2 \sqrt{\frac{m_{e}}{\hbar^{2}}} \sqrt{2 \bar{W}}} \ln \left(1-r \frac{1}{e V} \frac{L_{0}}{\rho_{b}+L_{0}} \frac{\hbar^{2}}{2 m^{*}} \frac{1}{x} \mathrm{e}^{-2 \frac{x}{L_{\phi}}}\right. \\
& \left.\quad \times\left(\frac{\xi_{k_{\mathrm{eV}}}}{\sinh \xi_{k_{\mathrm{eV}}}} k_{\mathrm{eV}} J_{1}\left(2 k_{\mathrm{eV}} x\right)-\frac{\xi_{k_{\mathrm{F}}}}{\sinh \xi_{k_{\mathrm{F}}}} k_{\mathrm{F}} J_{1}\left(2 k_{\mathrm{F}} x\right)\right)\right)+s_{0} .
\end{aligned}
$$

By using Eq. (13) as starting point, we neglected the energy and bias voltage dependence of the transmission factor. Numerical solution of the integral in Eq. (19) with $\rho_{\text {step }}(E, x)$ from Eq. $(18), \mathscr{T}(E, V, s)$ from Eq. (20) and parameters in the range of those used in our experiment yield, that neglecting the energy and bias dependence of $\mathscr{T}(E, V, s)$ is very well justified in the low-bias regime which is the subject of our interest here ( $V$ typically $10 \mathrm{mV}$ ). Using Eq. (24) we have three free parameters for fitting, namely the effective step reflection amplitude $r, L_{\phi}$ and the step location. $k_{\mathrm{eV}}$ is known from the dispersion relations, $\bar{W}$ from apparent barrier height measurements and $L_{0} /\left(\rho_{\mathrm{b}}+L_{0}\right)$ from spectra on clean terraces [45].

According to Eq. (24) the damping of quantum interference patterns as measured in constant-current mode of STM is caused by a combination of inelastic scattering processes $\left(\mathrm{e}^{-2 x / L_{\phi}}\right)$, FermiDirac broadening $\left(\xi_{k_{\mathrm{ev}}} / \sinh \xi_{k_{\mathrm{eV}}}\right)$ and beating due to the fact that $k$ vectors from $k_{\mathrm{F}}$ up to $k_{\mathrm{eV}}$ contribute to the current and thus to $\left.s(x)\right|_{I, V}(1 / \mathrm{eV}$ and $1 / x)$. To compare the damping strength of these different contributions we define, in addition to $L_{\phi} / 2$ for inelastic processes, the following characteristic apparent damping constants. The expression of FermiDirac broadening $\xi_{k} / \sinh \xi_{k}$ takes on the value $1 / e$ at $\xi_{k} \approx 2.7$, this defines (see Eq. (14))

$L_{\mathrm{FD}} \approx 2.7 \frac{\hbar^{2}}{2 \pi m^{*}} \frac{k_{\mathrm{eV}}}{k_{\mathrm{B}} T}$.

The beating or wave vector spread due to summing over $k$ vectors from $k_{\mathrm{F}}$ up to $k_{\mathrm{eV}}$ leads to a damping over the characteristic length $L_{\mathrm{V}} \approx 1 / \Delta k$ with $\Delta k=$ $k_{\mathrm{eV}}-k_{\mathrm{F}}=\sqrt{2 m^{*} / \hbar^{2}}\left(\sqrt{e V-E_{\Gamma}^{-}}-\sqrt{-E_{\Gamma}^{-}}\right)$, or for small $V, \Delta k=\left(m^{*} e V / \hbar^{2} k_{\mathrm{F}}\right)$. Depending on the chosen conditions $(V, T)$ one of these three damping lengths is shortest and dominates the decay. Since we are interested in e-ph processes we would like to extract $L_{\phi}$ from our data, and therefore we aim to measure in a regime where $L_{\phi}<L_{\mathrm{FD}}, L_{\mathrm{V}}$. Contrary to $L_{\phi}$ and $L_{\mathrm{FD}}, L_{\mathrm{V}}$ is temperature independent and by measuring at very low bias voltages $L_{\mathrm{V}}$ is virtually infinite and thus constitutes no major obstacle. The situation is different for $L_{\mathrm{FD}}$. Both $L_{\mathrm{FD}}$ and $L_{\phi}$ decrease with increasing temperature and $L_{\phi}$ will dominate the damping only if $\mathrm{e}-\mathrm{ph}$ coupling is strong enough, e.g. in the simple Debye model $\lambda \gtrsim$ $1 / 2.7 \approx 0.37^{9}$.

The validity of $\left.s(x)\right|_{I, V}$ of Eq. (24) was already demonstrated in Fig. 4(a). The dominant damping is due to $L_{\mathrm{FD}}$ in this case $\left(L_{\phi}=\infty\right.$ for the fit). The line scan in Fig. 4(b) has been taken at a bias voltage of $100 \mathrm{mV}$. At these conditions $L_{\mathrm{V}}$ prevails. The line scan shows the beating of the Bessel functions with wave vectors $k_{100 \mathrm{meV}}$ and $k_{\mathrm{F}}$.

\section{1. $\operatorname{Ag}(111)$}

In Fig. 9(a) line scans taken at $V=10 \mathrm{mV}$ from 3.5 $\mathrm{K}$ to $77.3 \mathrm{~K}$ on a $\operatorname{Ag}(111)$ terrace adjacent to a descending step are presented. The spatial damping of the standing waves with increasing $T$ is clearly visible. The line scans in Fig. 9(a) have been fitted by Eq. (24) for $x \geq 30 \AA$ and by putting $L_{\phi}=\infty$. The data and the fitted function coincide almost perfectly, except in the immediate vicinity of the step edge where the model is not valid. The spatial damping is dominated by $L_{\mathrm{FD}}$ at high $T$, and by $L_{\mathrm{V}}$ at low temperatures. It is clear from the fits that $L_{\phi} / 2>$ $L_{\mathrm{FD}}, L_{\mathrm{V}}$ in the experiment. The fit parameter $r_{\mathrm{desc}}=$ $0.56 \pm 0.06$ does not vary significantly with temperature and is in good agreement with reflection amplitudes determined in independent experiments [41].

Since the effective damping length $L_{\mathrm{FD}}$ due to Fermi-Dirac broadening is inversely proportional to $T$, at temperatures larger than $100 \mathrm{~K}$, constant-current line scans taken at low bias voltage across a

\footnotetext{
${ }^{9} L_{\mathrm{e}-\mathrm{ph}}$ is always larger than its high-temperature extrapolation [7], i.e. $L_{\mathrm{e}-\mathrm{ph}} \geq \hbar^{2} k_{\mathrm{eV}} / 2 \pi m^{*} \lambda k_{\mathrm{B}} T$ (Eq. (4)). Asking for $L_{\mathrm{e}-\mathrm{ph}}$ to be smaller than $L_{F D}$ from Eq. (25) leads to the condition $\lambda \geq 1 / 2.7$.
} 


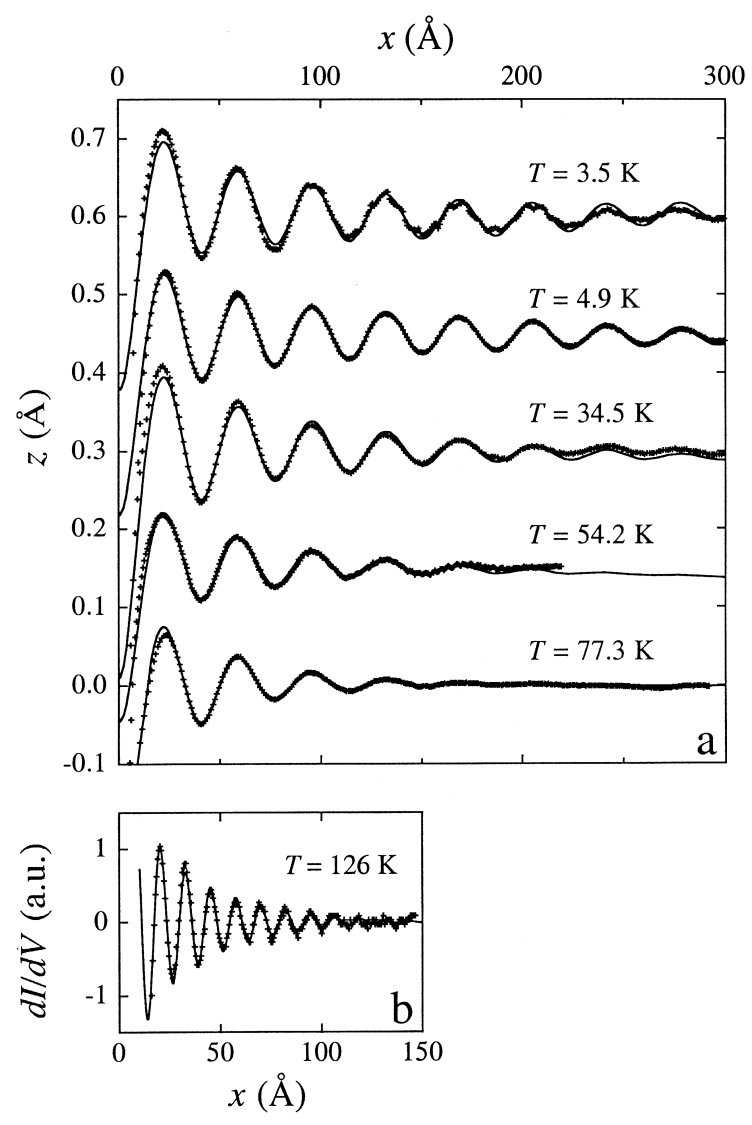

Fig. 9. (a) $\operatorname{Ag}(111)$ constant-current line scans taken on a terrace adjacent to a descending step $(V=10 \mathrm{mV}, I=1.0 \mathrm{nA})$. The data have been displaced vertically for clarity. (b) $\mathrm{d} I / \mathrm{d} V$ data taken across a step at $V=403 \mathrm{mV}, I=4.3 \mathrm{nA}$, and $T=126 \mathrm{~K}(\Delta V=79$ $\mathrm{mV}$, c.f., $\nu=5.37 \mathrm{kHz}$ ). The solid lines are fits using Eq. (24) in (a), and Eqs. (21) and (22) for $\mathrm{d} I / \mathrm{d} V$ in (b), with the reflectivity $r$ and the step location being the only fit parameters $\left(L_{\phi}\right.$ was set to infinity, see text).

$\operatorname{Ag}(111)$ step show too few oscillations for a significant fit procedure (Fig. 9(a)). However, since $L_{\mathrm{FD}}$ $\propto k_{\mathrm{eV}}$ (Eq. (25)) this problem can be circumvented by measuring quantities like $\mathrm{d} I / \mathrm{d} V$ or $\mathrm{d} z / \mathrm{d} V$ with lockin technique at larger bias voltage. At larger bias values $\mathrm{d} I / \mathrm{d} V$ rather than constant-current line scans are used since $L_{\Delta \mathrm{V}}$ from Eq. (23) is generally much smaller than $L_{\mathrm{V}}$. Fig. 9 (b) shows $\mathrm{d} I / \mathrm{d} V$ data across a step at $T=126 \mathrm{~K}$, acquired as described in Section 3. These data are perfectly fitted by Eqs. (21) and (22) and $L_{\phi}=\infty$. Thus, also at $126 \mathrm{~K} L_{\phi} / 2>L_{\mathrm{FD}}$.

Within our model the temperature dependent damping of the standing waves of the $s-p$ surface state on $\operatorname{Ag}(111)$ is very well described by the Fermi-Dirac broadening alone. Therefore we can only give lower limits for the phase-relaxation length $L_{\phi}$. For $\operatorname{Ag}(111)$ surface-state electrons $L_{\phi}$ is estimated to be $L_{\phi}\left(E_{\mathrm{F}}\right) \geqslant 600 \AA$ at $3.5 \mathrm{~K}$ and $L_{\phi}\left(E_{\mathrm{F}}\right) \geqslant$ $250 \AA$ at $77 \mathrm{~K}$. These limits are obtained by reducing $L_{\phi}$ in the fit function, Eq. (24), until a significant deviation from the experimental data is observed.

\section{2. $\mathrm{Cu}(111)$}

The $\mathrm{s}-\mathrm{p}$ surface state on $\mathrm{Cu}(111)$ shows a $k_{\mathrm{F}}$ that is larger than for $\operatorname{Ag}(111)$. Therefore, for a given temperature, $L_{\mathrm{FD}}\left(E_{\mathrm{F}}\right)$ is larger on $\mathrm{Cu}(111)$ than on $\operatorname{Ag}(111)$ (Eq. (25)). At the Fermi energy FermiDirac broadening is hence expected to play a smaller role for $\mathrm{Cu}(111)$ than for $\mathrm{Ag}(111)$. This explains why we can clearly observe standing waves in lowbias constant-current images on $\mathrm{Cu}(111)$ up to room temperature, whereas on $\operatorname{Ag}(111)$ no waves are observable at $300 \mathrm{~K}$ in such images (see also Ref. [59] for standing waves at $300 \mathrm{~K}$ on $\mathrm{Cu}$ ). Furthermore, beating effects (i.e. $L_{\mathrm{V}}$ ) should also be smaller for $\mathrm{Cu}(111)$ due to its steeper dispersion in the vicinity of $E_{\mathrm{F}}$. Our results of the temperature induced spatial damping on the $\mathrm{Cu}(111)$ surface represented in Fig. 10 are as for $\operatorname{Ag}(111)$ fully reproduced by Eq. (24) assuming $L_{\phi}=\infty$. Again, there is perfect agreement between model and experiment and the observed apparent coherence loss can entirely be

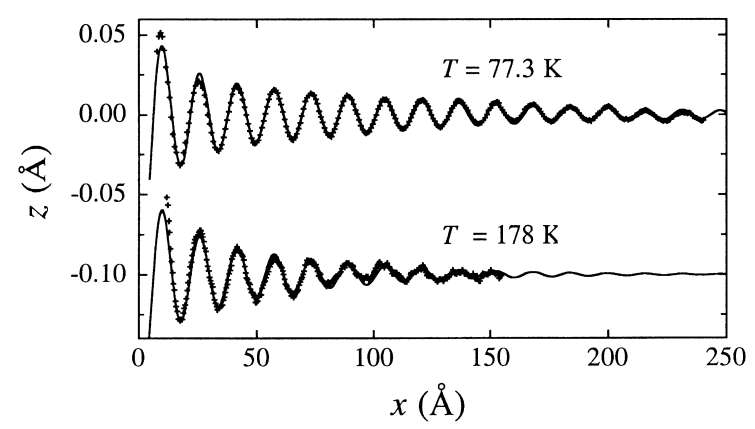

Fig. 10. $\mathrm{Cu}(111)$ constant-current line scans taken at $V=10 \mathrm{mV}$ on a terrace adjacent to a descending step. The data have been displaced vertically for clarity $(I=0.4 \mathrm{nA}$ at $77.3 \mathrm{~K}, I=0.1 \mathrm{nA}$ at $178 \mathrm{~K})$. The solid lines are fits using Eq. (24) $\left(L_{\phi}\right.$ was set to infinity, see text). 
explained in the framework of Fermi-Dirac broadening. As in the case of $\operatorname{Ag}(111)$ the lower limits of $L_{\phi}$ for $\mathrm{Cu}(111)$ are obtained by reducing $L_{\phi}$ in the fit function Eq. (24) until a significant deviation from the data is observed. The estimates are $L_{\phi}\left(E_{\mathrm{F}}\right) \geqslant 660$ $\AA$ at $77 \mathrm{~K}$ and $\gtrsim 160 \AA$ at $178 \mathrm{~K}$ (see Fig. 11).

\subsection{Discussion}

Photoemission lines originating from surface states are preferred candidates for electron lifetime studies since surface states have no dispersion with respect to $\mathbf{k}_{\perp}$. Hence the instrumental final state uncertainty in that quantity does not lead to broadening, and the linewidth $\Gamma$ gives direct access to the lifetime broadening of the initial state [16]. The currently
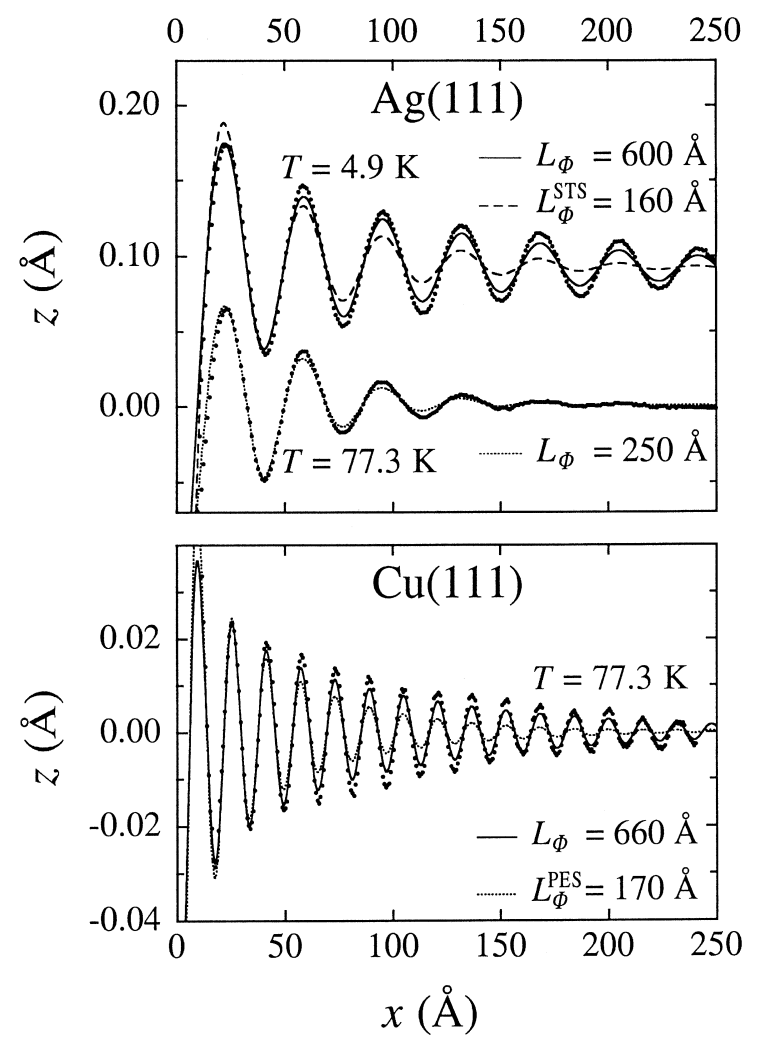

Fig. 11. Decay of standing waves as determined in experiment for $\mathrm{Ag}(111)$ and $\mathrm{Cu}(111)$ compared to results from Eq. (24) employing various values for the phase coherence length $L_{\phi}$. It is clearly seen that the values deduced in former STS and ARPES studies are too small compared to our experimental result. (For a detailed discussion of, and references to $L_{\phi}^{\mathrm{STS}}$ and $L_{\phi}^{\mathrm{PES}}$ see text.) most accurate photoemission studies of surface-state linewidths have been reported by McDougall et al. [7] and Matzdorf et al. [10] for $\mathrm{Cu}(111)$, and by Paniago et al. [60] for $\operatorname{Ag}(111)$. From the $T$ dependence of $\Gamma$, McDougall et al. could derive the electron-phonon interaction strength of the $\mathrm{s}-\mathrm{p}$ derived surface state on $\mathrm{Cu}(111)\left(\Gamma_{\mathrm{e}-\mathrm{ph}}=2 \pi \lambda k_{\mathrm{B}} T\right.$, Eq. (4)). Their result of $\lambda=0.14 \pm 0.02$ was experimentally confirmed by Matzdorf et al. [10] and agrees well with theory $(\lambda=0.15 \pm 0.03)[6]$. Despite the remarkable success of high-resolution photoemission to infer $\lambda$ from $d \Gamma / d T$, the absolute linewidths $\Gamma$ reported so far are all far above the theoretical predictions. This deficiency of PES is well known; it could be attributed to broadening by scattering at substrate imperfections [10,20,61]. In agreement with this interpretation, $\mathrm{Li}$ et al. report in a recent STS study on $\operatorname{Ag}(111)$ an unprecedented small $\Gamma$ value from local measurements on surface areas that were bare of defects [37].

We compare inverse lifetimes derived from STS and ARPES with our measurements of the decay of standing waves in Fig. 11. For the sake of comparison we converted all quantities in $L_{\phi}=v_{\mathrm{F}} \tau_{\phi}=$ $v_{\mathrm{F}} \hbar / \Gamma$, where $v_{\mathrm{F}}$ is the group velocity of the electrons at our measuring energy $E_{\mathrm{F}}$. From the width of the onset of the $\operatorname{Ag}(111)$ surface state in tunneling spectra taken at $5 \mathrm{~K}, \mathrm{Li}$ et al. derived $\tau_{\phi}\left(E_{\Gamma}^{-}\right)=67 \pm 8$ fs corresponding to $L_{\phi}^{\mathrm{STS}}=$ $v_{\mathrm{F}} \tau_{\phi}\left(E_{\Gamma}^{-}\right) \approx 160 \AA$ [37]. It is evident from Fig. 11 that this result gives too large decay rates as compared to our $\operatorname{Ag}(111)$ data taken at $E_{\mathrm{F}}$ and $4.9 \mathrm{~K}$. The shorter lifetime observed at $\bar{\Gamma}$ is probably partly due to the fact that the electron-phonon linewidth levels off at low temperature at the $\bar{\Gamma}$ point [7], and partly due to $\mathrm{e}-\mathrm{e}$ interaction, which of course is enhanced at the $\bar{\Gamma}$ point as compared to $E_{\mathrm{F}}$. It is also seen that our lower bound of $L_{\phi}\left(E_{\mathrm{F}}\right)=600 \AA$ is conservative, presumably $L_{\phi}\left(E_{\mathrm{F}}\right)$ is much larger. Our $L_{\phi}$ value presents the largest lifetime measured so far for the $\operatorname{Ag}(111)$ surface state. It corresponds to a peak width of $\Gamma\left(E_{\mathrm{F}}, 3.5 \mathrm{~K}\right)=\left(\hbar^{2} k_{\mathrm{F}} / m^{*} L_{\phi}\right) \lessgtr 2.6$ $\mathrm{meV}$, which should be very difficult to resolve in ARPES.

In the Debye model the phase-relaxation length due to electron-phonon interaction close to $E_{\mathrm{F}}$ is described by $L_{\mathrm{e}-\mathrm{ph}}\left(E_{\mathrm{F}}, T\right)=v_{\mathrm{F}} / \tau_{\mathrm{e}-\mathrm{ph}}\left(E_{\mathrm{F}}, T\right)$, where $\tau_{\mathrm{e}-\mathrm{ph}}\left(E_{\mathrm{F}}, T\right)$ is obtained through numerical integra- 
tion of Eq. (3) with $\lambda$ being the only free parameter ${ }^{10}$. Since there is only one parameter, an absolute measurement of $L_{\phi}\left(E_{\mathrm{F}}\right)$ at a single $T$ yields an estimate of $\lambda$. We have used the Debye model of Eq. (3) to derive such estimates from our $L_{\phi}\left(E_{\mathrm{F}}\right)$ values. For $\mathrm{Ag}\left(\hbar \omega_{\mathrm{D}}^{\mathrm{Ag}}=19 \mathrm{meV}\right.$ [5] $)$ we derive $\lambda_{\mathrm{Ag}} \lesssim 0.27$ from $L_{\phi}\left(E_{\mathrm{F}}, 77.3 \mathrm{~K}\right) \gtrsim 250 \AA$. This conservative upper limit for the electron-phonon mass enhancement factor is in agreement with the bulk value of $\lambda_{\mathrm{Ag}}=0.13 \pm 0.04$ given by Grimwall [6].

The currently lowest intrinsic linewidth measured by PES for the $\bar{\Gamma}$ surface state on $\mathrm{Cu}(111)$ is $\Gamma\left(E_{\Gamma}^{-}, 77 \mathrm{~K}\right)=36 \mathrm{meV}[7]$. By deducing the difference of electron-phonon and electron-electron linewidths between $E_{\Gamma}^{-}$and $E_{\mathrm{F}}$ of $\Delta \Gamma=8 \mathrm{meV}+5 \mathrm{meV}=$ $13 \mathrm{meV}$ (values inferred from the Debye model in Eq. (3) for $\lambda=0.14$ and from Eq. (1)), we estimate the resulting ARPES linewidth at $E_{\mathrm{F}}$ to be about $\Gamma\left(E_{\mathrm{F}}, 77 K\right)=23 \mathrm{meV}$, respectively, $L_{\phi}^{\mathrm{PES}}\left(E_{\mathrm{F}}, 77 \mathrm{~K}\right) \approx$ $170 \AA$. Fig. 11 shows that this coherence length again is considerably too short compared to the observed decay length of the standing waves. Our lower bound of $L_{\phi}\left(E_{\mathrm{F}}, 77 \mathrm{~K}\right)=660 \AA$ yields a linewidth of $\Gamma\left(E_{\mathrm{F}}, 77 \mathrm{~K}\right)=6 \mathrm{meV}$. From this upper bound of $\Gamma\left(E_{\mathrm{F}}, 77 \mathrm{~K}\right)$, and consistently from our measurement at $178 \mathrm{~K}\left(\Gamma\left(E_{\mathrm{F}}, 178 \mathrm{~K}\right) \lesssim 26 \mathrm{meV}\right)$, we derive an upper limit of $\lambda_{\mathrm{Cu}} \lesssim 0.34$ using the Debye model of Eq. (3) with $\hbar \omega_{\mathrm{D}}^{\mathrm{Cu}}=27 \mathrm{meV}$. Again this is a conservative estimate which is in accordance with $\lambda=0.14$ measured with ARPES [7,10].

One evident reason why we measure much larger coherence lengths than can possibly be obtained with photoelectron spectroscopy is that we determine $L_{\phi}$ locally at terrace stripes perpendicular to steps that are bare of any adsorbates or other steps on the length scale of $L_{\phi}$. From large scale observations of the surface morphology it is clear, however, that every crystal presents surface areas where the average terrace width is below our $L_{\phi}$ values. Also, at a lot of surface spots the density of chemical defects is above $1 / L_{\phi}^{2}$, for $L_{\phi}$ in the range discussed here. Every integrating technique will be embarrassed by the steps and point defects since surface-state elec-

\footnotetext{
${ }^{10}$ In this model we assume that the surface state electrons couple to phonons in the same way as bulk electrons do. Furthermore, surface phonons are not considered.
}

trons strongly couple to the bulk at these sites, i.e. are scattered out of the surface state [41]. This leads to an apparent reduction of the integral $L_{\phi}$ as seen in PES. We therefore believe that the 'offset' of $\Gamma(0 \mathrm{~K}) \approx 20-30 \mathrm{meV}$ characterizing high-resolution PES peaks is mainly due to structural defects such as steps $[7,10]$. This assignment is supported by differences of up to $10 \mathrm{meV}$ in the linewidth 'offset' between different research groups, whereas there is good agreement on $d \Gamma / d T[7,10]$. The influence of sputter defects on the linewidth $\Gamma$ was employed to extrapolate to 'intrinsic' linewidths expected from PES of perfectly ordered surfaces [61]. The resulting 'intrinsic' values, e.g., of $\Gamma\left(E_{\Gamma}^{-}, 0 \mathrm{~K}\right) \leq 21 \pm 5 \mathrm{meV}$ for $\mathrm{Cu}(111)$, still contain phonon excitation at $0 \mathrm{~K}$ and electron-electron interactions.

The alternative approach to look at STS peak widths eliminates the defect problem, however, the analysis in terms of lifetimes demands elaborate modeling. We note that our STS peak widths [45] are comparable to the ones reported by $\mathrm{Li}$ et al. [37], hence we would infer similar estimates on $\tau$ from regarding $\Gamma\left(E_{\Gamma}^{-}, 5 \mathrm{~K}\right)$ in our STS spectra. Compared to a peak width analysis our access to the e-ph part of $\tau_{\phi}$ via measuring $L_{\phi}$ from the decay of standing waves has two advantages, i) it is based on a straightforward analytical model that has been tested experimentally, and ii) since we measure at $E_{\mathrm{F}}$, our $L_{\phi}$ values are not reduced by electron-electron scattering and therefore provide a more direct access to $\lambda$.

To conclude, we point out that in contrast to integral measurements such as photoemission we measure the phase-relaxation length $L_{\phi}$ locally. This eliminates residual linewidths due to surface defect scattering embarrassing integrating techniques. Our STM-results therefore provide currently the best absolute estimates of $L_{\phi}$, respectively inelastic lifetime $\tau_{\phi}=L_{\phi} / v_{\mathrm{F}}$ for the $\mathrm{s}-\mathrm{p}$ surface states on $\mathrm{Cu}(111)$ and $\operatorname{Ag}(111)$. In principle, by the technique described in this Section, STM constitutes a powerful method to study e-ph interactions at surfaces. Since e-ph interaction in $\mathrm{Cu}$ and $\mathrm{Ag}$ with mass enhancement parameters of $\lambda_{\mathrm{Cu}}=0.15$ and $\lambda_{\mathrm{Ag}}=$ 0.13 is relatively weak [6], the technique is embarrassed by the fact that $L_{\mathrm{FD}}<L_{\mathrm{e}-\mathrm{ph}}$, and therefore we have not been able to determine an absolute value of the e-ph interaction strength in these systems, but only an upper limit. In future studies, by choosing 
systems with an e-ph mass enhancement parameter $\lambda \gtrsim 0.4$, STM could be used to quantify electronphonon interactions at surfaces. Metallic elements with relatively large e-ph interaction include [6]: $\mathrm{Al}$, Ti, V, Nb, Ta, Mo, W, Re, Ir, Pd, Pb, Sn.

\section{References}

[1] S. Datta, Electronic Transport in Mesoscopic Systems, Cambridge University Press, Cambridge, 1995.

[2] J.W. Gadzuk, Phys. Rev. Lett. 76 (1996) 4234.

[3] D. Pines, P. Nozières, The Theory of Quantum Liquids, Benjamin, New York, 1966.

[4] J.J. Quinn, Physical Review 126 (1962) 1453.

[5] N.W. Ashcroft, N.D. Mermin, Solid State Physics, Saunders College, Philadelphia, 1976.

[6] G. Grimvall, The Electron-Phonon Interaction in Metals, North-Holland, New York, 1981.

[7] B.A. McDougall, T. Balasubramanian, E. Jensen, Phys. Rev. B 51 (1995) 13891.

[8] H. Petek, H. Nagano, S. Ogawa, Phys. Rev. Lett. 83 (1999) 832.

[9] D. Purdie, M. Hengsberger, M. Gamier, Y. Baer, Surf. Sci. 407 (1998) L671.

[10] R. Matzdorf, G. Meister, A. Goldmann, Phys. Rev. B 54 (1996) 14807.

[11] A. Yacoby, U. Sivan, C.P. Umbach, J.M. Hong, Phys. Rev. Lett. 66 (1991) 1938.

[12] S.Q. Murphy, J.P. Eisenstein, L.N. Pfeiffer, K.W. West, Phys. Rev. B 52 (1995) 14825.

[13] G.F. Giuliani, J.J. Quinn, Phys. Rev. B 26 (1982) 4421.

[14] L. Zheng, S. Das Sarma, Phys. Rev. B 53 (1996) 9964.

[15] A. Goldmann, R. Matzdorf, F. Theilmann, Surf. Sci. 414 (1998) L932.

[16] R. Matzdorf, Surf. Sci. Rep. 30 (1998) 153.

[17] S.D. Kevan, R.H. Gaylord, Phys. Rev. B 36 (1987) 5809.

[18] R. Paniago, R. Matzdorf, G. Meister, A. Goldmann, Surf. Sci. 331-333 (1995) 1233.

[19] S.D. Kevan, Phys. Rev. Lett. 50 (1983) 526.

[20] J. Tersoff, S.D. Kevan, Phys. Rev. B 28 (1983) 4267.

[21] C.A. Schmuttenmaer, M. Aeschlimann, H.E. Elsayed-Ali, R.J.D. Miller, Phys. Rev. B 50 (1994) 8957.

[22] M. Aeschuimann, M. Bauer, S. Pawlik, Chemical Physics 205 (1996) 127.

[23] T. Hertel, E. Knoesel, M. Wolf, G. Ertl, Phys. Rev. Lett. 76 (1996) 535.

[24] E. Knoesel, A. Hotzel, T. Hertel, M. Wolf, G. Ertl, Surf. Sci. 368 (1996) 76.

[25] S. Xu, J. Cao, C.C. Miller, D.A. Mantell, R.J.D. Miller, Y. Gao, Phys. Rev. Lett. 76 (1996) 483.

[26] W. Wallauer, T. Fauster, Surf. Sci. 374 (1997) 44.

[27] S. Ogawa, H. Nagano, H. Petek, Phys. Rev. B 55 (1997) 10869.

[28] E. Knoesel, A. Hotzel, M. Wolf, Phys. Rev. B 57 (1998) 12812.
[29] M.F. Crommie, C.P. Lutz, D.M. Eigler, Nature 363 (1993) 524.

[30] Y. Hasegawa, P. Avouris, Phys. Rev. Lett. 71 (1993) 1071.

[31] M.F. Crommie, C.P. Lutz, D.M. Eigler, Science 262 (1993) 218.

[32] P. Avouris, J.-W. Lyo, Science 264 (1994) 942.

[33] P. Avouris, I.-W. Lyo, R.E. Walkup, Y. Hasegawa, J. Vac. Sci. Technol. B 12 (3) (1994) 1447.

[34] S. Crampin, M.H. Boon, J.E. Inglesfield, Phys. Rev. Lett. 73 (1994) 1015.

[35] J. Li, W.-D. Schneider, R. Berndt, S. Crampin, Phys. Rev. Lett. 80 (1998) 3332.

[36] S. Crampin, O.R. Bryant, Phys. Rev. B 54 (1996) R17367.

[37] J. Li, W.-D. Schneider, R. Berndt, O.R. Bryant, S. Crampin, Phys. Rev. Lett. 81 (1998) 4464.

[38] J. Li, W.-D. Schneider, S. Crampin, R. Berndt, Surf. Sci. 422 (1999) 95.

[39] P. Avouris, J.-W. Lyo, P. Molinàs-Mata, Chem. Phys. Lett. 240 (1995) 423.

[40] G. Hörmandinger, J.B. Pendry, Phys. Rev. B 50 (1994) 18607.

[41] L. Bürgi, O. Jeandupeux, A. Hirstein, H. Brune, K. Kern, Phys. Rev. Lett. 81 (1998) 5370.

[42] A. Selloni, P. Carnevali, E. Tosatti, C.D. Chen, Phys. Rev. B 31 (1985) 2602.

[43] N.D. Lang, Phys. Rev. B 34 (1986) 5947.

[44] I. Adawi, Phys. Rev. 146 (1966) 379.

[45] O. Jeandupeux, L. Bürgi, A. Hirstein, H. Brune, K. Kern, Phys. Rev. B 59 (1999) 15926.

[46] J.J. Paggel, T. Miller, T.-C. Chiang, Science 283 (1999) 1709.

[47] E.J. Heller, M.F. Crommie, C.P. Lutz, D.M. Eigler, Nature 369 (1994) 464.

[48] J. Tersoff, D.R. Hamann, Phys. Rev. Lett. 50 (1983) 1998.

[49] G. Hörmandinger, Phys. Rev. B 49 (1994) 13897.

[50] R. Smoluchowski, Phys. Rev. 60 (1941) 661.

[51] J. Li, W.-D. Schneider, R. Berndt, Phys. Rev. B 56 (1997) 7656.

[52] L. Bürgi, L. Petersen, H. Brune, K. Kern, Surf. Sci. 447 (2000) 157.

[53] C. Kittel, Introduction to Solid State Physics, John Wiley, New York, 1986.

[54] M. Rocca, L. Yibing, F. Buatier de Mongeot, U. Valbusa, Phys. Rev. B 52 (1995) 14947.

[55] I. Campillo, J.M. Pitarke, A. Rubio, E. Zarate, P.M. Echenique, Phys. Rev. Lett. 83 (1999) 2230.

[56] P.M. Echenique, Private communication.

[57] M. Schmid, W. Hebenstreit, P. Varga, S. Crampin, Phys. Rev. Lett. 76 (1996) 2298.

[58] L. Petersen, P. Laitenberger, E. Lægsgaard, F. Besenbacher, Phys. Rev. B 58 (1998) 7361.

[59] E. Wahlström, I. Ekvall, H. Olin, L. Wallden, Appl. Phys. A 66 (1998) S1107.

[60] R. Paniago, R. Matzdorf, G. Meister, A. Goldmann, Surf. Sci. 336 (1995) 113.

[61] F. Theilmann, R. Matzdorf, G. Meister, A. Goldmann, Phys. Rev. B 56 (1997) 3632. 